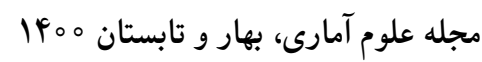

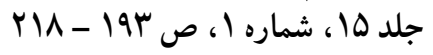

DOI: $10.29252 /$ jss.15.1.193

مقاله بُروهشى

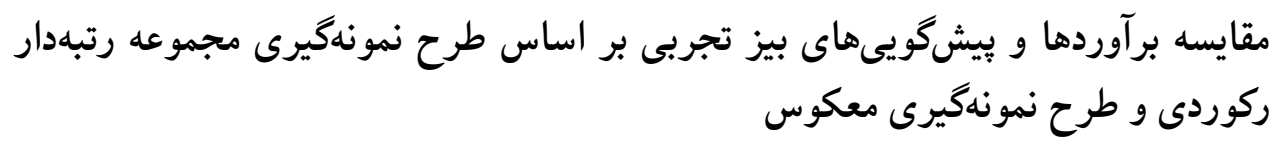

احسان گلزاده گروى'، برويز نصيرى '، سيدمهدى صالحى '

آخروه آمار، دانشكاه بيام نور، تهران

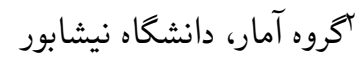

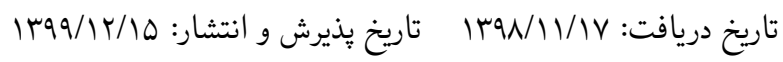

جكيده: در اين مقاله برآورد بيز تجربى يارامتر توزيع نمايى تحت توابع زيان توان دوم خطا و لاينكس

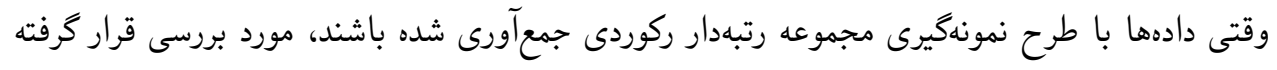

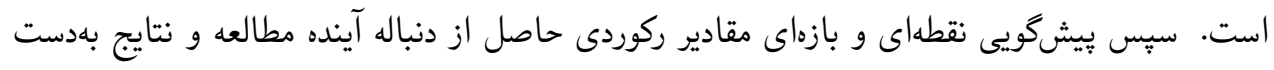

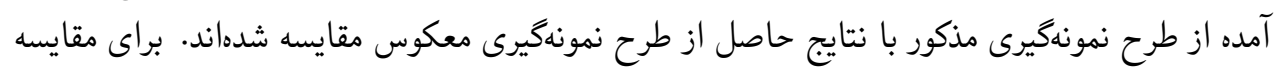

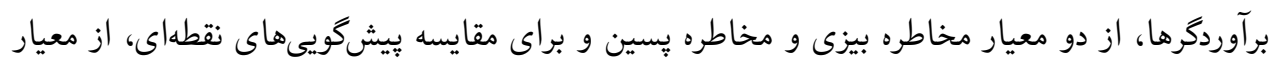

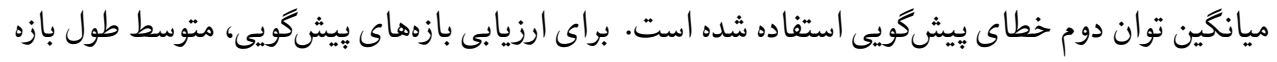

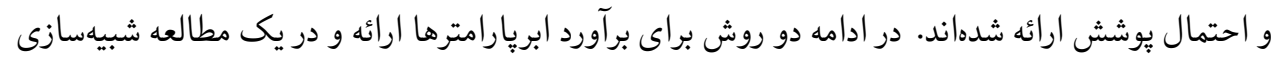

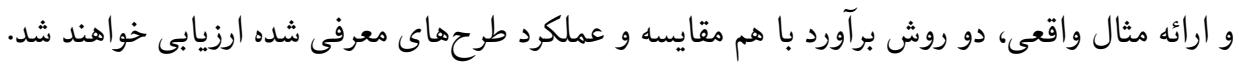
وازههاى كليدى: برآورد، ييشگويى، ركورد، بيز تجربى، نمونهيرى مجموعه رتبهدار ركوردى. 


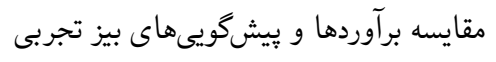

\section{|}

در يك طرح نمونهيرى با رهيافت بيزى برخلاف رهيافت كلاسيك، بارامتر مورد بررسى تحققى از يك توريك

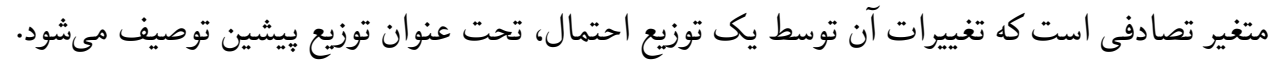

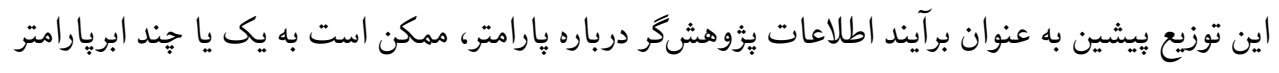

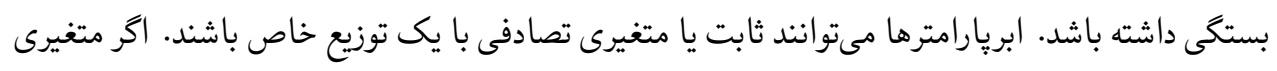

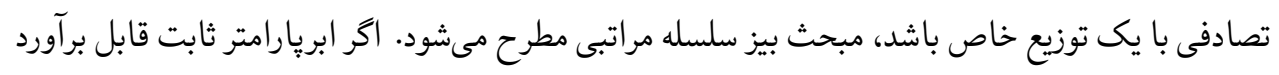

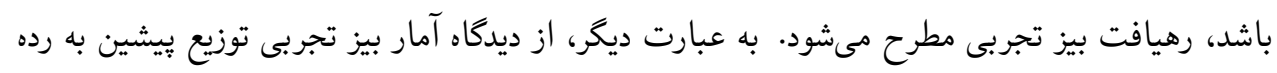

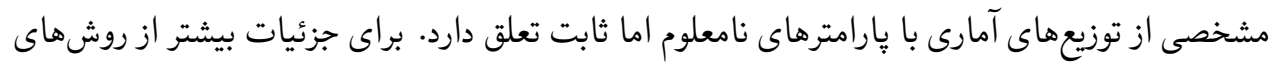

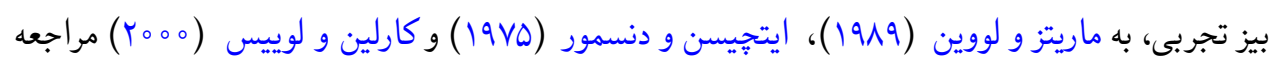

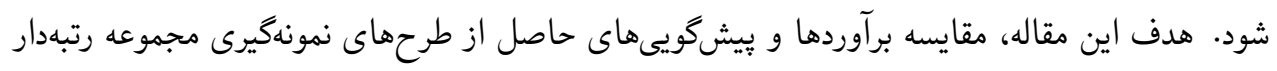

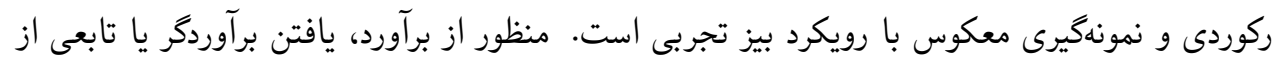

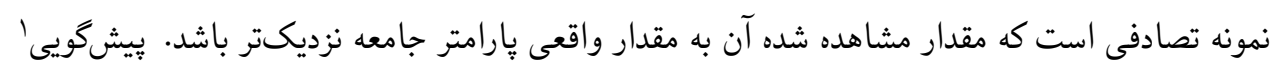

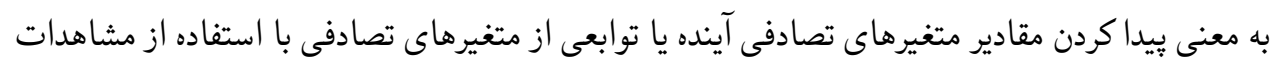

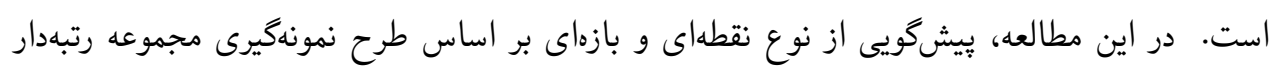

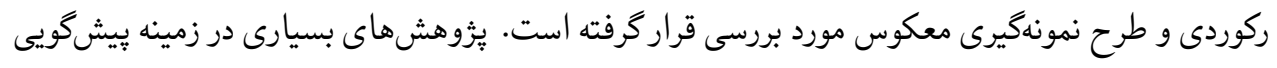

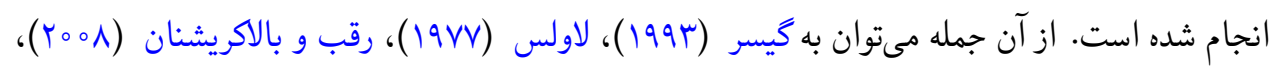

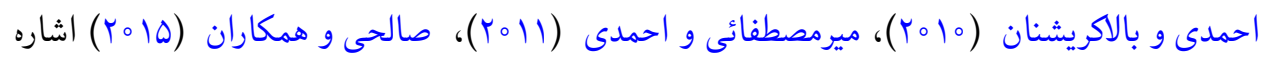

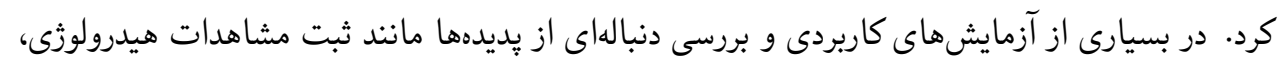

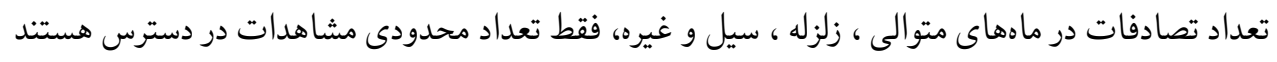

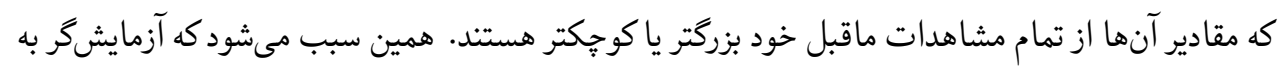

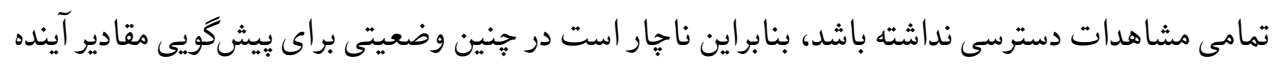

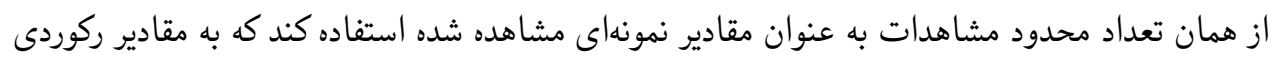

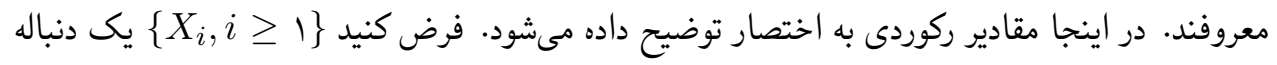

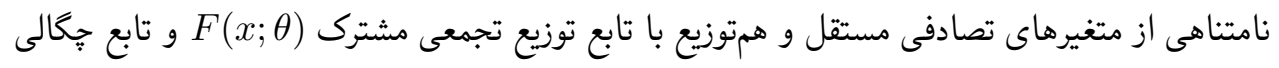

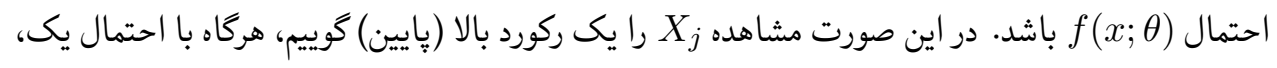
براى همه مقادير $i$ كه $i$ > 
است و $\}$ اسر إدت

$$
\text { يارامترى است بهطورى كه }
$$

$$
f(x ; \theta)=\theta e^{-\theta x}, \quad x>\circ, \theta>\circ .
$$

براى اطلاعات بيشتر درباره ركوردها به، احسناله (1990) ، آرنولد و همكاران (1991)، نوزوروف (1901)

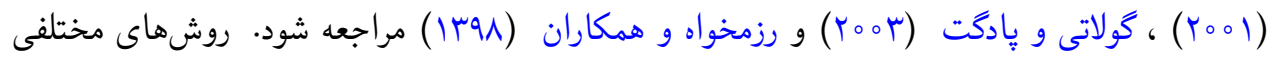

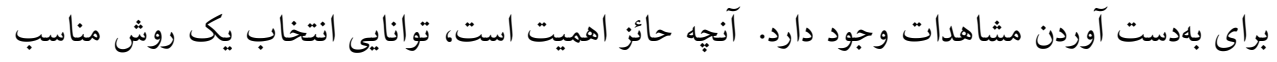

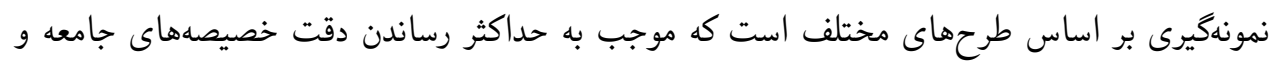

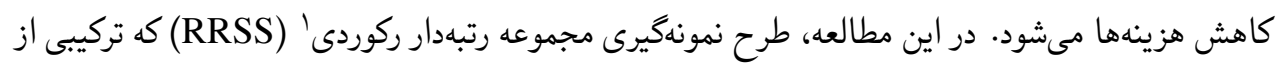

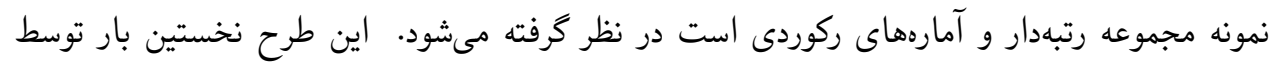

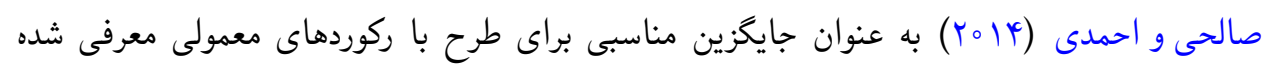

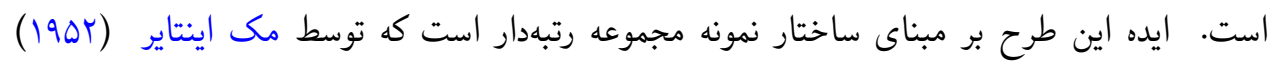

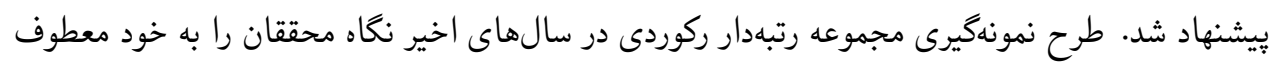

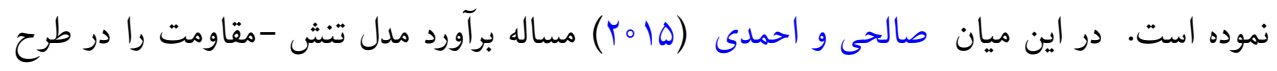

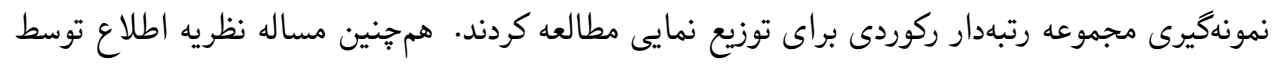

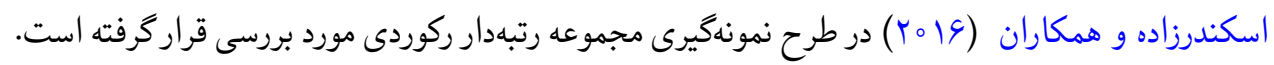

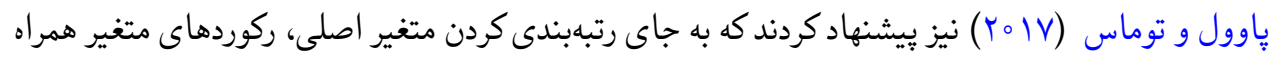

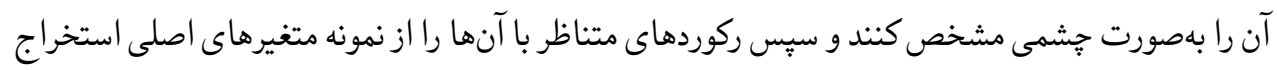

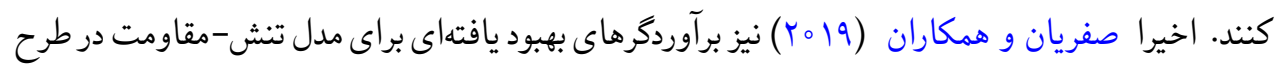

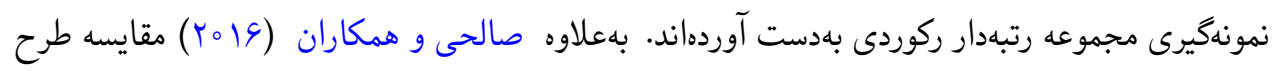

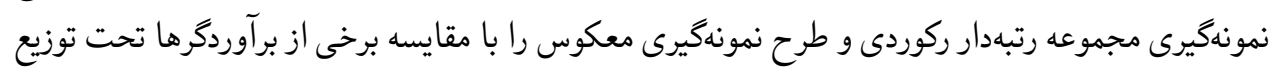

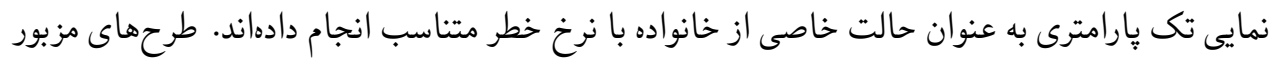

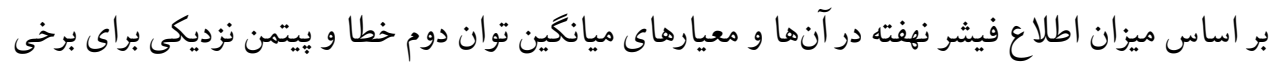

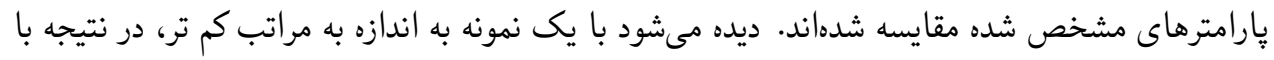

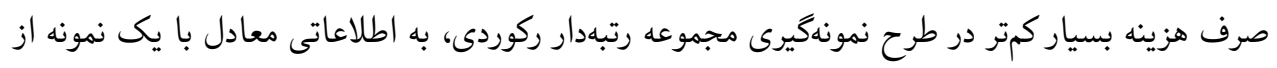

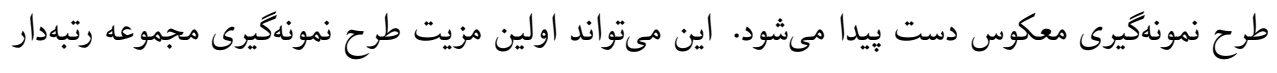


مقايسه برآوردها و يیشگويىهاى بيز تجربى

ركوردى نسبت به طرح نمونهكيرى معكوس باشد · به عنوان مزيت دوم مىتوان كفت ، ميزان اطلاعات

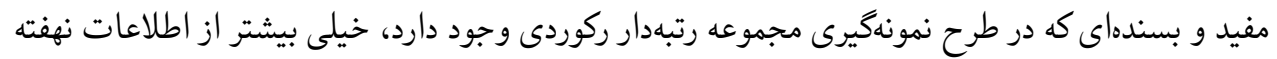

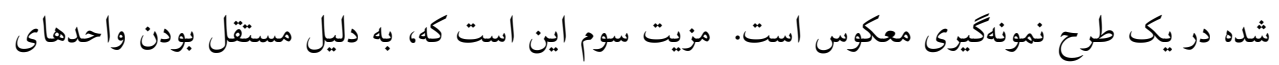

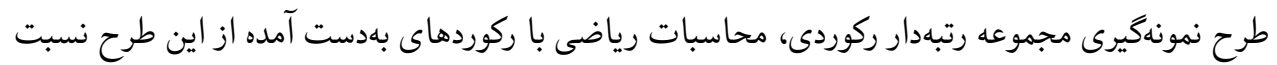

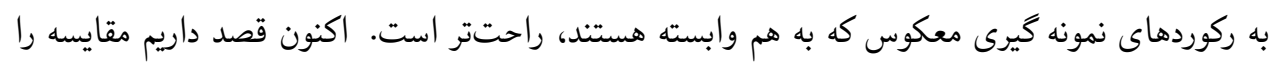

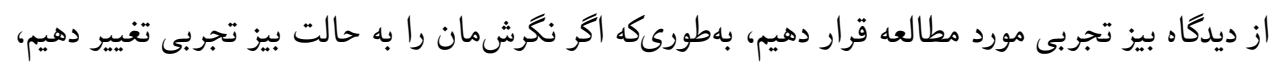

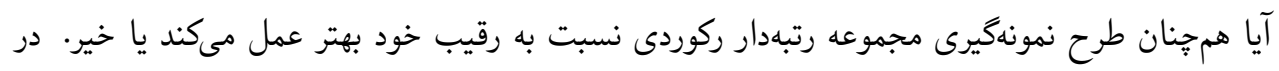

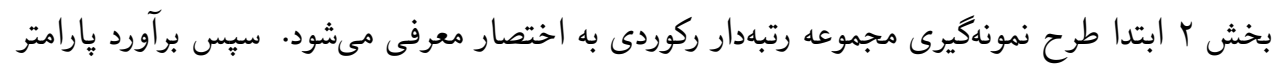

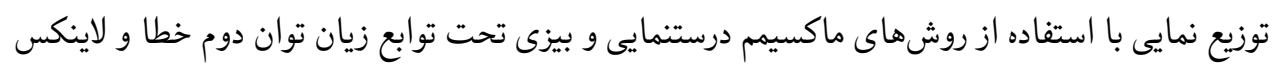

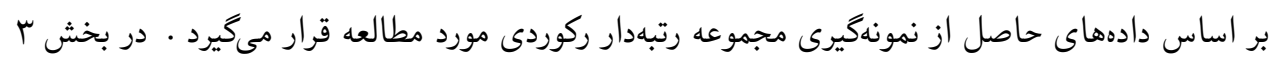

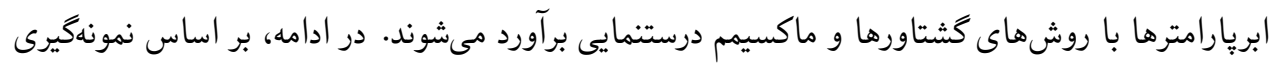

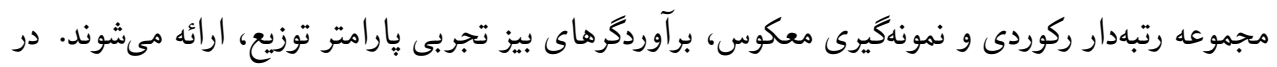

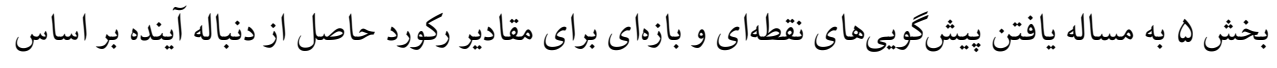

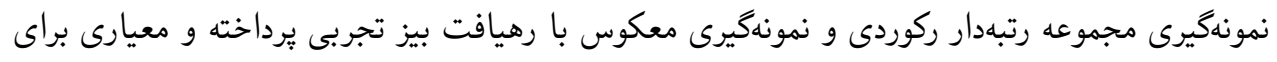

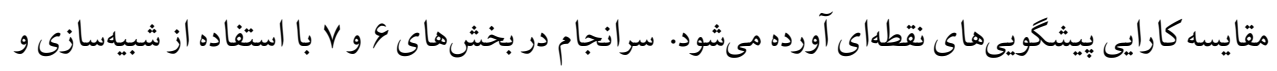

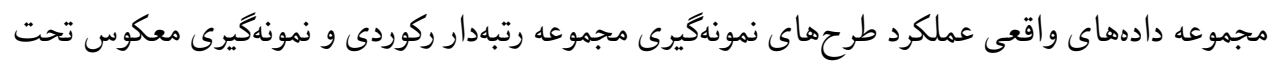

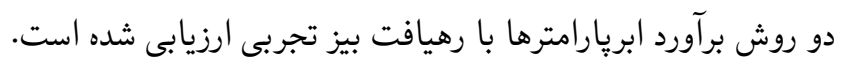

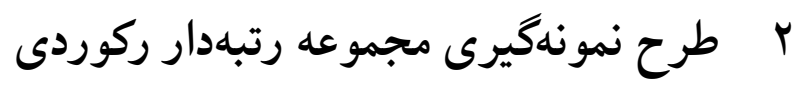

فرض كنيد n دنباله مستقل از متغيرهاى تصادفى در اختيار است و نمونهيرى در هر دنباله به نحوى است

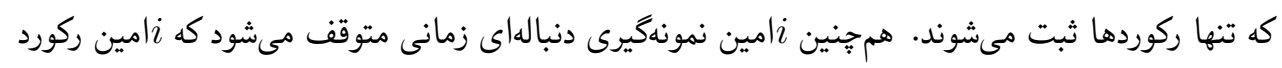

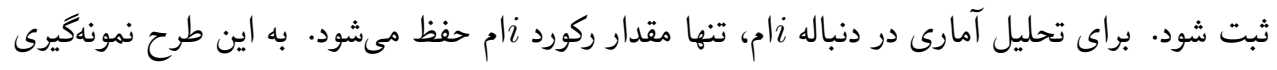

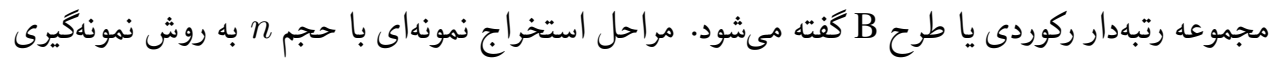
مجموعه رتبهدار ركوردى به كمك دياخرام 


$$
\begin{aligned}
& \text { 1: } R_{(1) 1} \\
& \rightarrow R_{1,1}=R_{(1) 1} \\
& r: R_{(1) r} \\
& \underline{R_{(r) r}} \\
& \rightarrow R_{\Upsilon, \digamma}=R_{(\Upsilon) \Upsilon} \\
& n: R_{(1) n} \\
& R_{(\Upsilon) n} \quad \cdots \quad \underline{R_{(n) n}} \rightarrow R_{n, n}=R_{(n) n}
\end{aligned}
$$

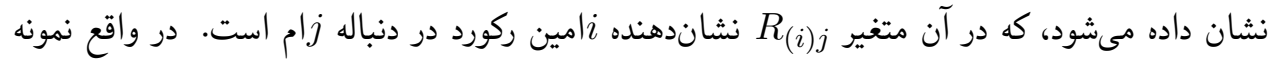

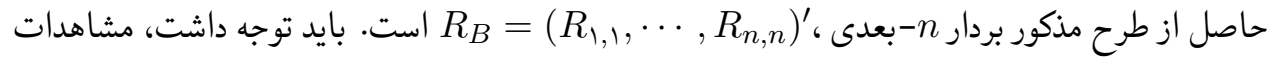

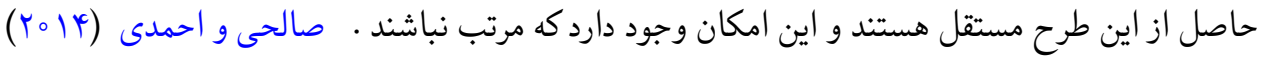

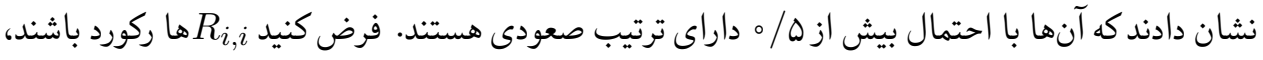

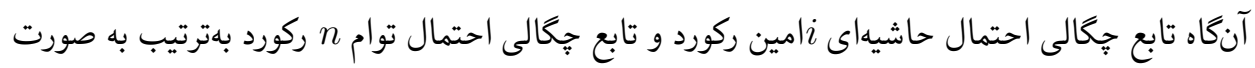

$$
\begin{aligned}
& f_{i, i}(x)=\frac{\{-\log \bar{F}(x)\}^{i-1}}{(i-1) !} f(x), \\
& f_{R_{B}}\left(r_{B} ; \theta\right)=\prod_{i=1}^{n} \frac{\left\{-\log \bar{F}\left(r_{i, i} ; \theta\right)\right\}^{i-1}}{(i-1) !} f\left(r_{i, i} ; \theta\right), \quad \theta \in \Theta,
\end{aligned}
$$

هستند، كه در آنها ${ }^{\prime}$ (. ف فضاى پارامتر است ( آرنولد و همكاران ، (1991).

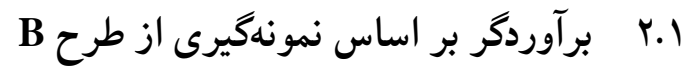

اخر (

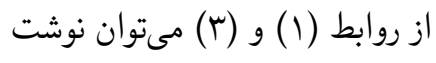

$$
L\left(\theta \mid r_{B}\right) \propto \theta^{N} e^{-\theta t}
$$


مقايسه برآوردها و بيشكويىهاى بيز تجربى

كه در آن درستنمايى $\theta$ به صورت

$$
\hat{\theta}_{M L E}(B) \equiv U=\frac{N}{T}
$$

است كه تابعى از آماره بسنده مينيمال كامل T است. با توجه به روابط (1) و (Y)، تابع جإكالى احتمال حاشيهاى iامين ركورد، پارامترهاى N و 0 است. مىتوان نشان داد،

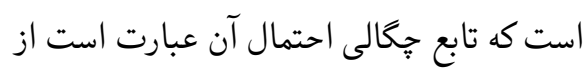

$$
f(u \mid \theta)=\frac{(N \theta)^{N}}{\Gamma(N)} u^{-N-1} e^{-\frac{N \theta}{u}}, u>\circ
$$

براى برآورد بيزى $\theta$ تابع زيان توان دوم خطاى باى

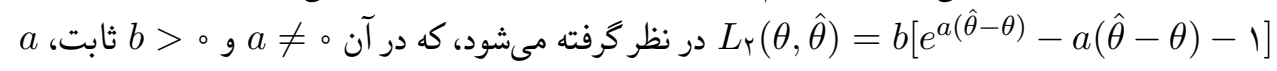

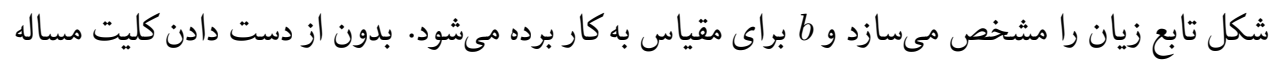

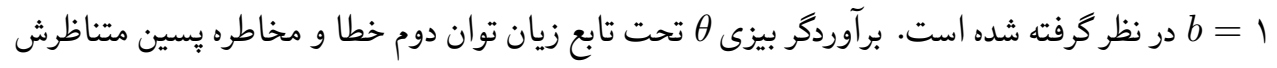
$r(\hat{\theta})=E\left(L(\theta, \hat{\theta}) \mid r_{B}\right)$ به ترتيب

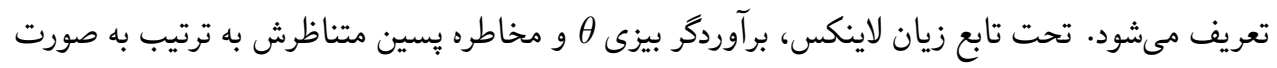

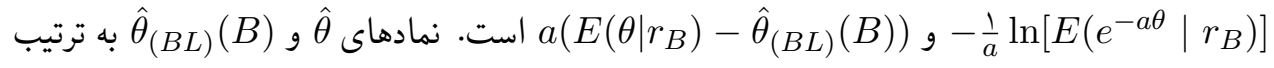
نشاندهنده برآوردخر يارامتر $\theta$ و برآوردكر بيزى $\theta$ تحت تابع زيان لاينكس بر اساس نمونهگيرى از طرح

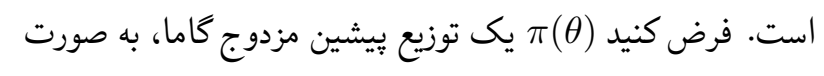

$$
\pi(\theta)=\frac{\beta^{\alpha}}{\Gamma(\alpha)} \theta^{\alpha-1} e^{-\beta \theta}, \quad \theta>\circ, \alpha>\circ, \beta>\circ
$$

باشد كه در آن م و م يارامترهاى توزيع يِيشين هستند و (·) تابع كاماى كامل است. با استفاده از روابط

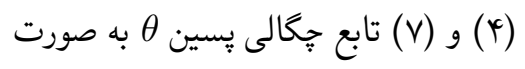

$$
\Pi\left(\theta \mid r_{B}\right) \propto \theta^{N+\alpha-1} e^{-\theta(\beta+t)},
$$


خواهد شد، به عبارت ديكر (A)

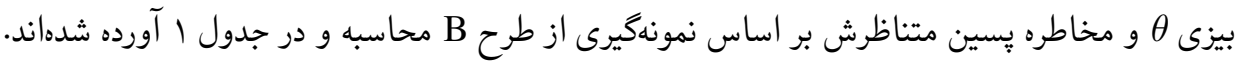

\begin{tabular}{|c|c|c|}
\hline مخاطره بֶسين & برآوردكر بيزى & تابع زيان \\
\hline$(N+\alpha)\left(\frac{a}{\beta+T}-\ln \left(1+\frac{a+\alpha}{\beta+T}\right)\right)$ & $\begin{array}{c}\quad \frac{N+\alpha}{\beta+T} \\
\frac{N+\alpha}{a} \ln \left(1+\frac{a}{\beta+T}\right)\end{array}$ & توان دوم خطا \\
\hline
\end{tabular}

\section{r م برآوردگ بيز تجربى بر اساس نموندگيرى از طرح}

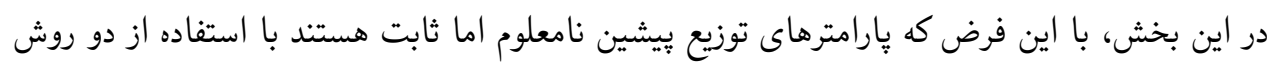

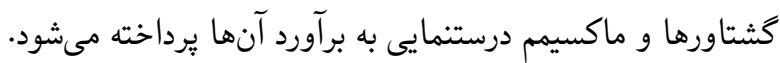

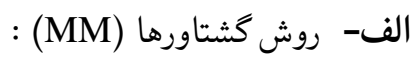
فرض كنيد بارامترهاى توزيع بيشين

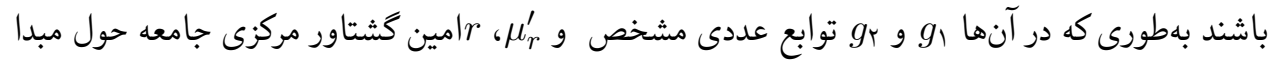

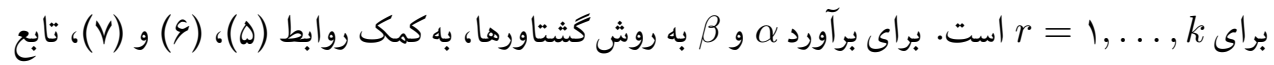

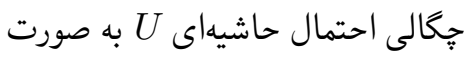

$$
\begin{aligned}
f(u) & =\int_{\circ}^{\infty} f(u \mid \theta) \pi(\theta) d \theta \\
& =\frac{1}{B(N, \alpha) u} q(u)^{N}(1-q(u))^{\alpha}, \quad u>\circ,
\end{aligned}
$$

حاصل مىشود، كه در آن مىشود ، برآوردهاى م و م به به روش گشتاورها به صورت

$$
\widetilde{\alpha_{\mathrm{B}}}=\frac{(N-1) q_{\uparrow}^{r}}{(N-r) q_{r}-(N-1) q_{\uparrow}^{r}}, \quad \widetilde{\beta_{\mathrm{B}}}=\frac{N q_{\uparrow}}{(N-r) q_{r}-(N-1) q_{\uparrow}^{r}},
$$


مقايسه برآوردها و ييشكوينهاى بيز تبربى

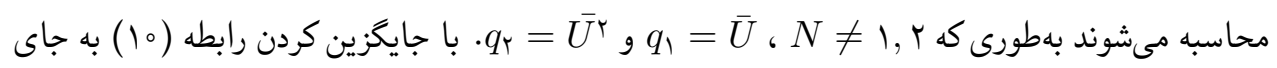

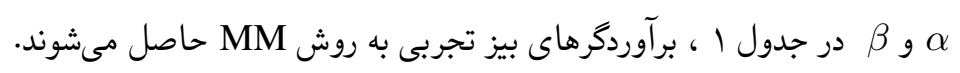

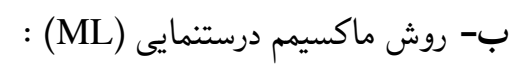
بر اساس نمونهيكي از طرح B ، ، با بكاركيرى رابطه (9) ميتوان نوشت $L\left(\alpha, \beta \mid u_{j}\right)=\prod_{j=1}^{m} \frac{1}{B(N, \alpha) u_{j}}\left(\frac{N}{N+\beta u_{j}}\right)^{N}\left(\frac{\beta u_{j}}{N+\beta u_{j}}\right)^{\alpha}, \quad u_{j}>0$.

به دنبال آن معادلات درستنمايى زير به شكل توابع غيرخطى كه فرم بستهاى ندارند حاصل مىشود

$$
\begin{aligned}
\frac{\partial \log L}{\partial \alpha} & =m(\Psi(N+\alpha)-\Psi(\alpha))+\sum_{j=1}^{m} \log \frac{\beta u_{j}}{N+\beta u_{j}}, \\
\frac{\partial \log L}{\partial \beta} & =\frac{m \alpha}{\beta}-(N+\alpha) \sum_{j=1}^{m} \frac{u_{j}}{N+\beta u_{j}},
\end{aligned}
$$

كه در آن

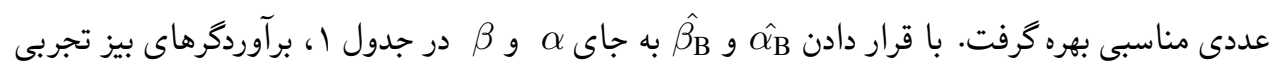

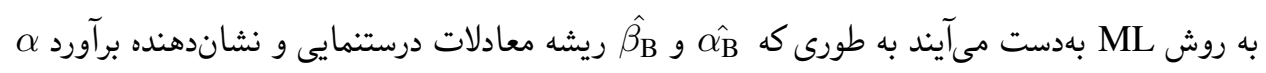

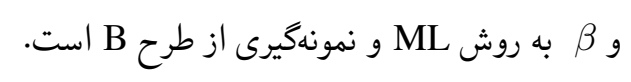

\section{ب برآوردكر بيز تجربى بر اساس طرح نمونهيرى معكوس}

فرض كنيد آزمايشها به صورت دنبالهاى انجام شده و نمونهكيرى زمانى خاتهه مي إبد كه ركورد n ام مشاهده

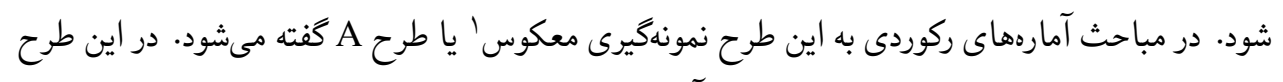

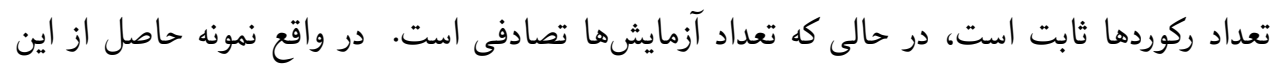

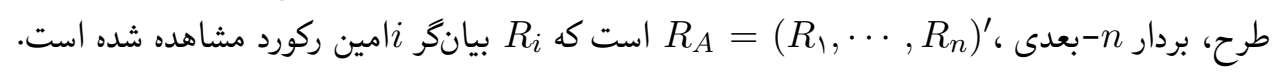

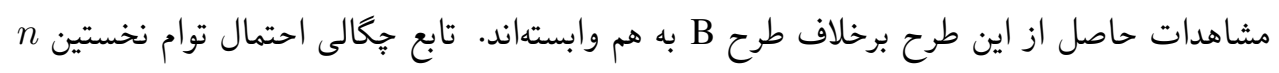

${ }^{1}$ Inverse Sampling Scheme 


$$
\text { ركورد اول به صورت } \left.f_{R_{A}}\left(r_{A} ; \theta\right)=f\left(r_{n} ; \theta\right) \prod_{i=1}^{n-1} \frac{f\left(r_{i} ; \theta\right)}{\bar{F}\left(r_{i} ; \theta\right)}, \quad r_{1}<\cdots<r_{n}, \quad \text { (I }\right)
$$

حاصل مىشود كه در آن ' A 1991). فرض كنيد (r)

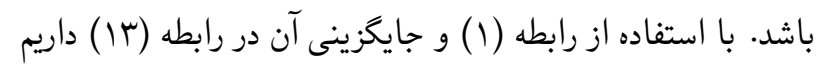

$$
L\left(\theta \mid r_{A}\right)=\theta^{n} e^{-\theta r_{n}}
$$

توزيع يسين براساس نمونهيرى از طرح A بر اساس روابط (V) و (Y ( ) به صورت زير حاصل خواهد شد

$$
\Pi\left(\theta \mid r_{A}\right)=\frac{\left(\beta+r_{n}\right)^{n+\alpha}}{\Gamma(n+\alpha)} \theta^{n+\alpha-1} e^{-\theta\left(\beta+r_{n}\right)}
$$

فرع ا. باكمى دقت مىتوان ديد ، اخر در نتايج طرح B به جاى (N، (T) به ترتيب از (n،

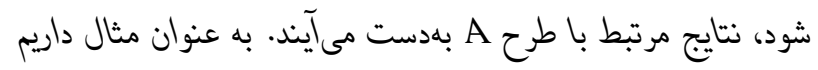

$$
\begin{aligned}
& \hat{\theta}_{M L E}(A) \equiv U^{\prime}=\frac{n}{R_{n}}, \\
& \widetilde{\alpha_{\mathrm{A}}}=\frac{(n-1) q_{1}^{\prime r}}{(n-r) q_{r}^{\prime}-(n-1) q_{\uparrow}^{r}}, \quad \widetilde{\beta_{\mathrm{A}}}=\frac{n q_{\uparrow}^{\prime}}{(n-r) q_{r}^{\prime}-(n-1) q_{\uparrow}^{\prime r}}, \\
& \text { كه در آنها }
\end{aligned}
$$

\section{ه بيشگيى بيز تجربى مقادير ركورد حاصل از دنباله آينده}

در اين بخش به مساله بيشكويى بيز تجربى مقادير ركورد حاصل از دنباله آينده برداخته مىشود. ابتدا بر

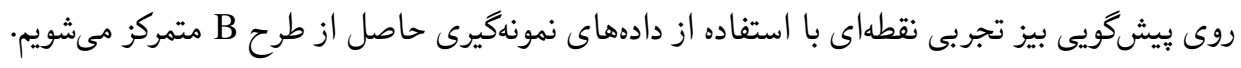




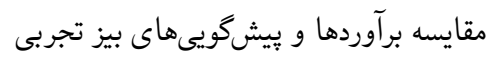

\section{B o.1}

فرض كنيد

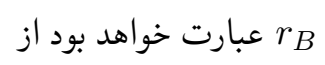

$$
f_{Y_{s}}^{*}\left(y \mid r_{B}\right)=\int_{\theta} f_{Y_{s}}(y \mid \theta) \Pi\left(\theta \mid r_{B}\right) d \theta
$$

با توجه به $Y_{S} \sim \operatorname{Gamma}(s, \theta$ و رابطه (^) خواهيم داشت

$$
f_{Y_{s}}^{*}\left(y \mid r_{B}\right)=\frac{1}{B(s, N+\alpha) y} p(y)^{s}(1-p(y))^{N+\alpha} y>\circ, s \geq 1,
$$

كه در آن

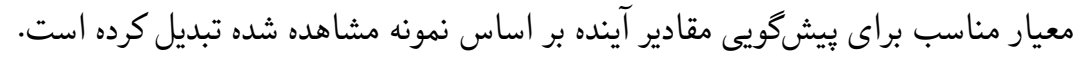

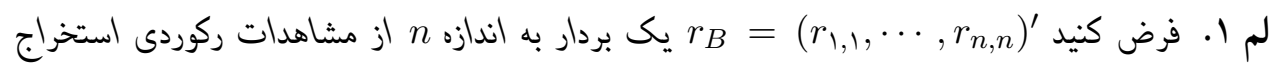

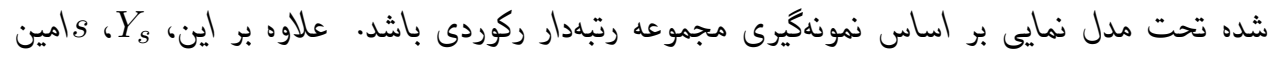

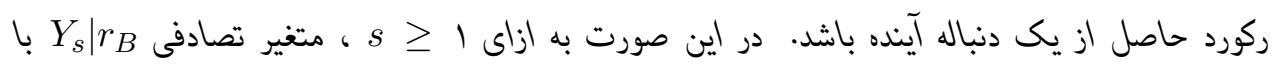

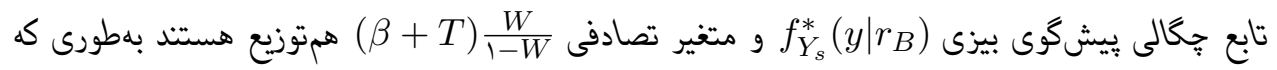
W 2 Beta $(s, N+\alpha)$

برهان: مىدانيم W يك متغير تصادفى بيوسته داراى توزيع بتا با تابع جآلى

$$
f(w)=\frac{1}{B(s, N+\alpha)} w^{s-1}(1-w)^{N+\alpha-1}, \quad \circ<w<1,
$$

است. فرض كنيد

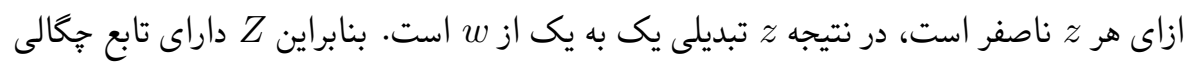

$$
\begin{aligned}
g(z) & =f\left(\frac{z}{\beta+t+z}\right) \frac{\beta+t}{(\beta+t+z)^{r}} \\
& =\frac{1}{B(s, N+\alpha) z} p(z)^{s}(1-p(z))^{N+\alpha}, \quad z>\circ .
\end{aligned}
$$


ror

احسان كلزاده گروى و همكاران .

خواهد بود كه همان رابطه (IV) است. در نتيجه لم فوق ثابت مىشود.

فرع r. مى مانيم، اخر

$\frac{N+\alpha}{s(\beta+T)} Y_{s} \mid r_{B} \sim F_{\curlyvee s, \Upsilon(N+\alpha)}$.

بيشگىى بيزى نقطهاى Y تحت تابع زيان توان دوم خطا، ميانخين توزيع بيشگو، يعنى

$$
\hat{Y}_{s}^{(B S)}=E\left(Y_{s} \mid r_{B}\right)=\frac{s}{N+\alpha-1}(\beta+T)
$$

است. ميانگين توان دوم خطاى اين بيشگو نيز به صورت

$$
\begin{aligned}
& E\left(\hat{Y}_{s}^{(B S)}-Y_{s}\right)^{r}=\frac{s^{r}}{(N+\alpha-1)^{r}}\left(\frac{N}{\theta^{r}}+\left(\beta+\frac{N}{\theta}\right)^{r}\right)-\frac{r s^{r}}{\theta(N+\alpha-1)}\left(\beta+\frac{N}{\theta}\right)+\frac{s}{\theta^{r}}+\frac{s^{r}}{\theta^{r}} . \\
& \text { به دست مىآيد. يُيشگويى بيزى نقطهاى Y تحت تابع زيان لاينكس نيز به صورت } \\
& \hat{Y}_{s}^{(B L)}=-\frac{1}{a} \ln E_{f^{*}}\left(e^{-a Y_{s}} \mid r_{B}\right) \\
& =-\frac{1}{a} \ln \sum_{k=0}^{\infty} \frac{\Gamma(k+s)}{\Gamma(s)} \frac{\Gamma(N+\alpha-k)}{\Gamma(N+\alpha)} \frac{(-a(\beta+T))^{k}}{k !},
\end{aligned}
$$

است. ميانگين توان دوم خطاى ييشگيى تحت تابع زيان لاينكس فرم بستهاى ندارد، لذا براى حل آن

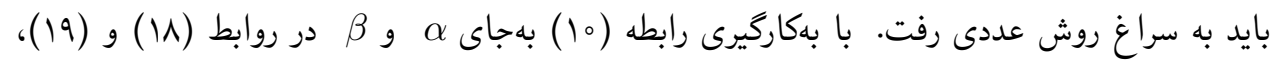

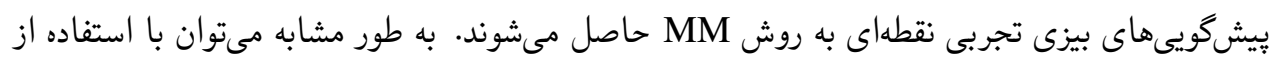

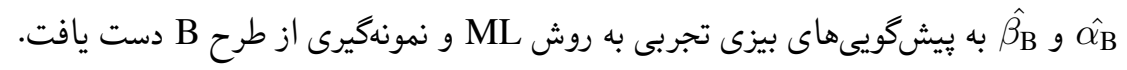


. . . مقايسه برآوردها و بيشكويىهاى بيز تجربى . . .

\section{B o.r}

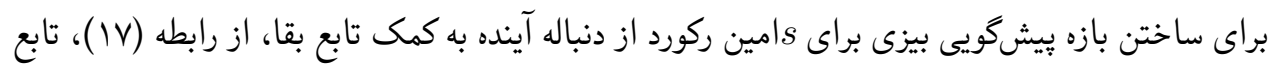

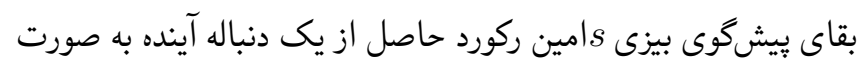

$$
\bar{F}_{Y_{s}}^{*}\left(y \mid r_{B}\right)=\int_{y}^{\infty} \frac{1}{B(s, N+\alpha) z} p(z)^{s}(1-p(z))^{N+\alpha} d z,
$$

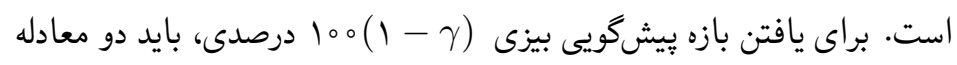

$$
\bar{F}_{Y_{s}}^{*}\left(L\left(r_{B}\right)\right)=1-\frac{\gamma}{r}, \quad \bar{F}_{Y_{s}}^{*}\left(U\left(r_{B}\right)\right)=\frac{\gamma}{r},
$$

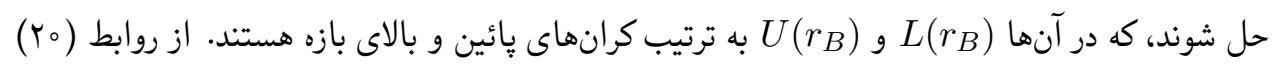

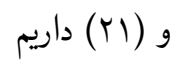

$$
\begin{aligned}
& \int_{0}^{L\left(r_{B}\right)} \frac{1}{B(s, N+\alpha) z} p(z)^{s}(1-p(z))^{N+\alpha} d z=\frac{\gamma}{r}, \\
& \text { با تغيير متغير } \\
& \int_{0}^{\left(1+\frac{\beta+t}{L\left(r_{B}\right)}\right)^{-1}} \frac{1}{B(s, N+\alpha)} v^{s-1}(1-v)^{N+\alpha-1} d v=\frac{\gamma}{r},
\end{aligned}
$$

با استفاده از رابطه (T) بهسادكى خواهيم داشت

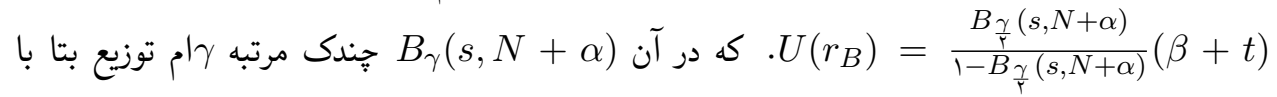

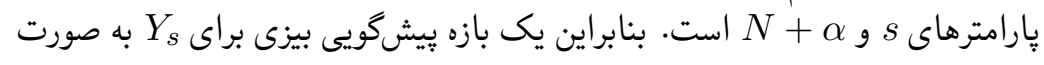

$$
\left(\frac{B_{1-\frac{\gamma}{r}}(s, N+\alpha)}{1-B_{1-\frac{\gamma}{r}}(s, N+\alpha)}(\beta+T), \frac{B_{\frac{\gamma}{r}}(s, N+\alpha)}{1-B_{\frac{\gamma}{r}}(s, N+\alpha)}(\beta+T)\right),
$$


حاصل مىشود. اين يك بازه تصادفى است. لذا متوسط طول بازه بيشكويى $=\left(\frac{N}{\theta}+\beta\right)\left\{\frac{B_{\frac{\gamma}{\gamma}}(s, N+\alpha)-B_{1-\frac{\gamma}{r}}(s, N+\alpha)}{\left(1-B_{\frac{\gamma}{\gamma}}(s, N+\alpha)\right)\left(1-B_{1-\frac{\gamma}{r}}(s, N+\alpha)\right)}\right\}$.

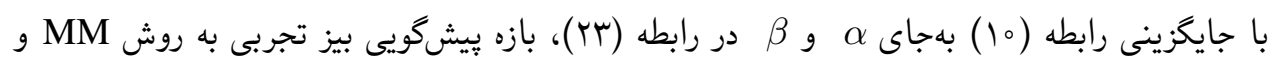

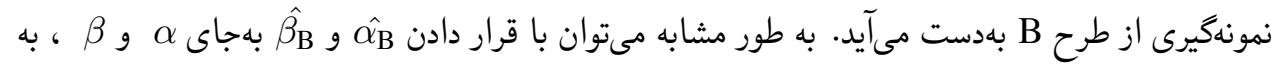

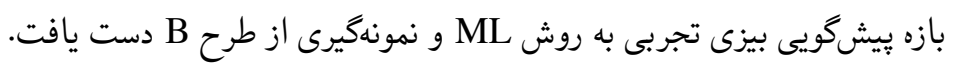

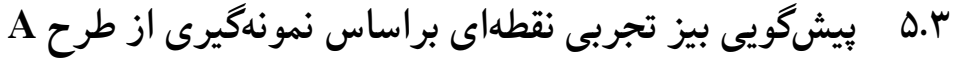

فرض كنيد،

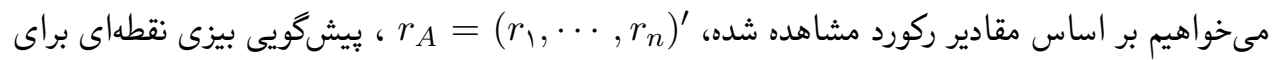

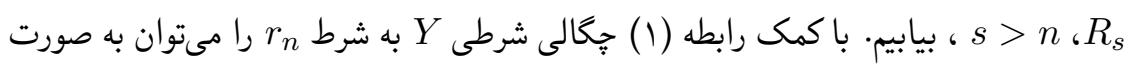

$$
\begin{aligned}
& g\left(y \mid r_{n}\right)=\frac{\left[\ln \frac{1-F\left(r_{n}\right)}{1-F(y)}\right]^{s-n-1}}{(s-n-1) !} \frac{f(y)}{\bar{F}\left(r_{n}\right)}, \\
& =\frac{\left[y-r_{n}\right]^{s-n-1}}{(s-n-1) !} \theta^{s-n} e^{-\theta\left(y-r_{n}\right)}, \quad \circ<r_{n}<y<\infty . \\
& \text { نوشت. از طرفى تابع جگالى بيزى بِيشوى Y به شرط r به صورت } \\
& g^{*}\left(y \mid r_{A}\right)=\int_{\theta} g\left(y \mid r_{n}\right) \Pi\left(\theta \mid r_{A}\right) d \theta, \\
& \text { تعريف مىشود كه با استفاده از روابط (ها) و (YY) به فرم } \\
& g^{*}\left(y \mid r_{A}\right)=\frac{(\beta+y)^{-(s+\alpha)}}{B(s-n, n+\alpha)}\left(\beta+r_{n}\right)^{n+\alpha}\left(y-r_{n}\right)^{s-n-1}, \quad \circ<r_{n}<y,
\end{aligned}
$$


.... مقايسه برآوردها و ييشكويىهاى بيز تجربى

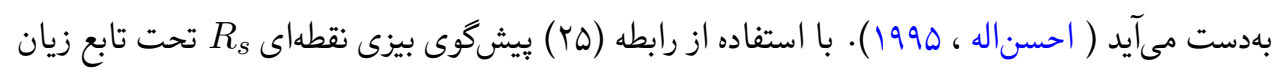

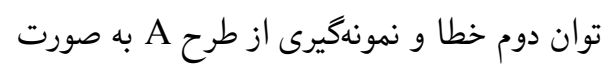

$$
\hat{R}_{s}^{(A S)}=\int_{r_{n}}^{\infty} y g^{*}\left(y \mid r_{A}\right) d y=\frac{s+\alpha-1}{n+\alpha-1}\left(\beta+r_{n}\right)-\beta, \quad s>n,
$$

است. معيار ميانكين توان دوم خطاى اين ييشكو نيز به صورت

$$
\begin{aligned}
E\left(\hat{R}_{s}^{(A S)}-R_{s}\right)^{r} & =\beta^{r}+\frac{r s \beta}{\theta}+\left(\frac{s+\alpha-1}{n+\alpha-1}\right)^{r}\left(\frac{n}{\theta^{r}}+\left(\beta+\frac{n}{\theta}\right)^{r}\right) \\
& -\curlyvee \beta\left(\frac{s+\alpha-1}{n+\alpha-1}\right)\left(\beta+\frac{n+s}{\theta}+\frac{n}{\beta \theta^{r}}(s+1)\right)+\frac{s}{\theta^{r}}+\frac{s^{r}}{\theta^{r}}
\end{aligned}
$$

است. همجنين ييشكويى بيزى نقطهاى تحت تابع زيان لاينكس و نمونهيرى از طرح A به صورت

$$
\hat{R}_{s}^{(A L)}=-\frac{1}{a} \ln \int_{r_{n}}^{\infty} e^{-a y} g^{*}\left(y \mid r_{A}\right) d y
$$

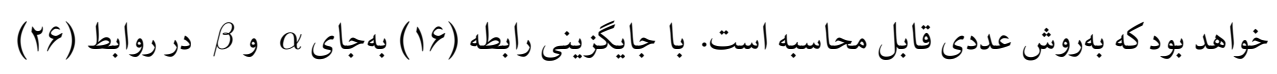

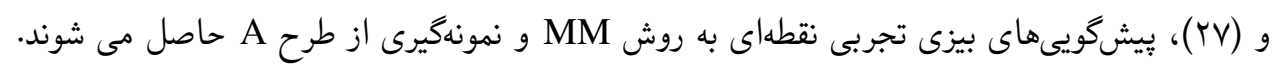

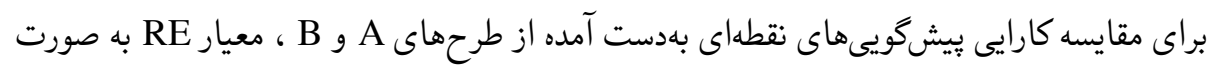

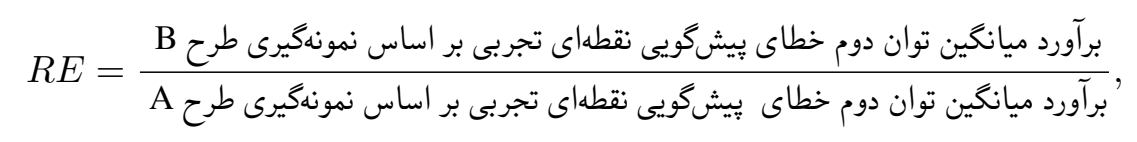

تعريف مىشود. اخر معيار RE كمتر از يك باشد، گواه محكمترى براى عملكرد بهتر طرح B است.

\section{A بيش ه.F}

با توجه به رابطه (YD)، مشابه طرح B ، بازه ييشكويى بيزى بر اساس نمونهكيرى از طرح A براى sامين

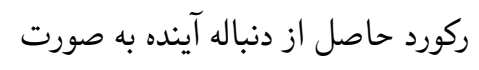

$$
\left(\frac{\beta+R_{n}}{B_{\frac{\gamma}{\Gamma}}(s-n, n+\alpha)}-\beta, \frac{\beta+R_{n}}{B_{\uparrow-\frac{\gamma}{r}}(s-n, n+\alpha)}-\beta\right)
$$




$$
\text { متوسط طول بازه بيش گيشويى }=\left(\frac{n}{\theta}+\beta\right)\left\{\frac{B_{\frac{\gamma}{r}}(s-n, n+\alpha)-B_{1-\frac{\gamma}{r}}(s-n, n+\alpha)}{B_{\frac{\gamma}{r}}(s-n, n+\alpha) B_{1-\frac{\gamma}{r}}(s-n, n+\alpha)}\right\} \text {. }
$$

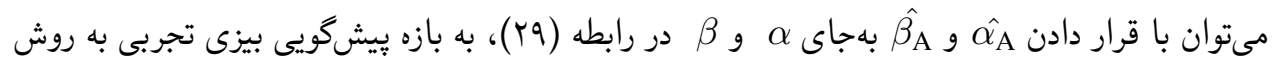

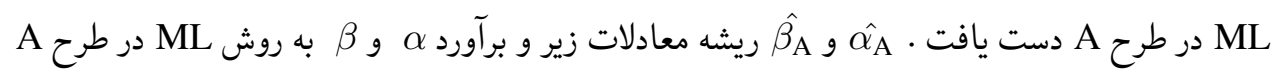

$$
\begin{aligned}
& \frac{\partial \log L}{\partial \alpha}=m(\Psi(n+\alpha)-\Psi(\alpha))+\sum_{j=1}^{m} \log \frac{\beta u_{j}^{\prime}}{n+\beta u_{j}^{\prime}}, \\
& \frac{\partial \log L}{\partial \beta}=\frac{m \alpha}{\beta}-(n+\alpha) \sum_{j=1}^{m} \frac{u_{j}^{\prime}}{n+\beta u_{j}^{\prime}} .
\end{aligned}
$$

\section{9 مطالعه شبيهسازى}

در اين بخش براى ارزيابى دو روش برآورد ابريارامترها ، مقايسه عملكرد طرحهاى معرفى شده و محاسبه

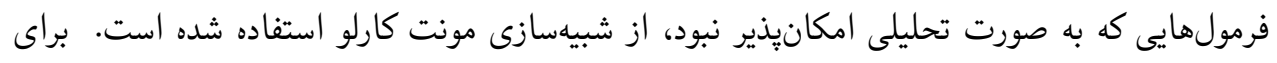

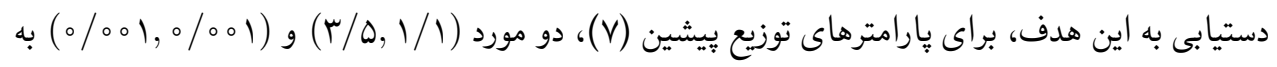

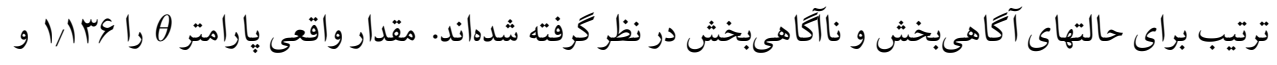

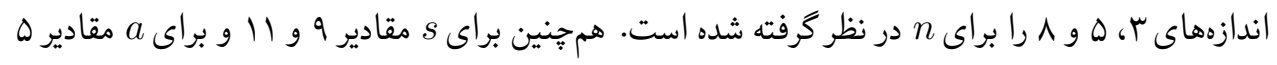

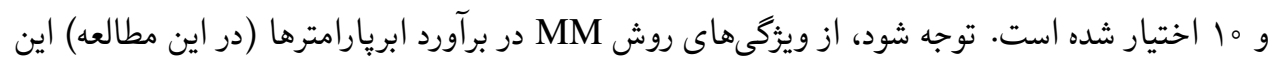

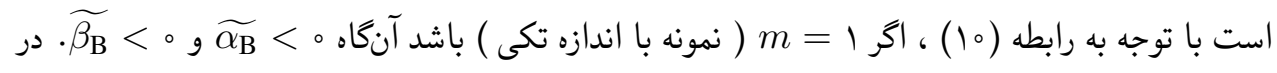

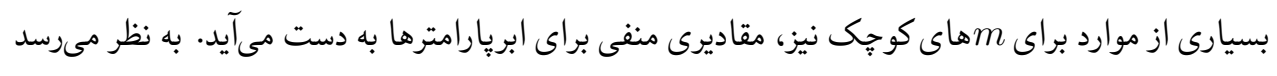

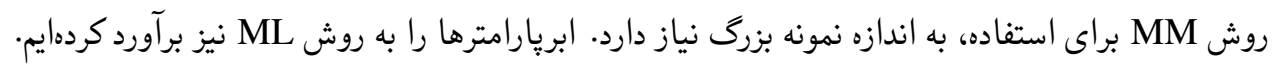

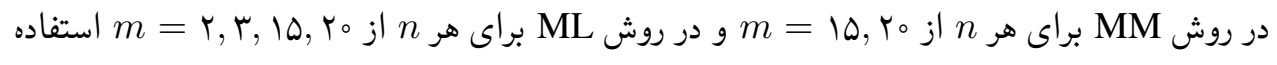

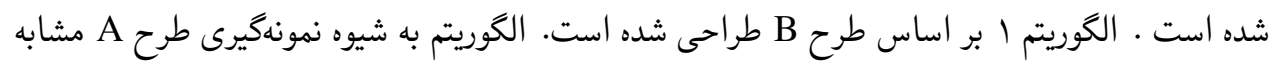
طرح B است.

الكوريتم I. بر اساس طرح B : 
مقايسه برآوردها و ييشكويىهاى بيز تجربى

كام ا- براى مقدار داده شده $\alpha$ و $\beta$ ، با استفاده از توزيع ييشين (V))، $\theta$ توليد مىشود.

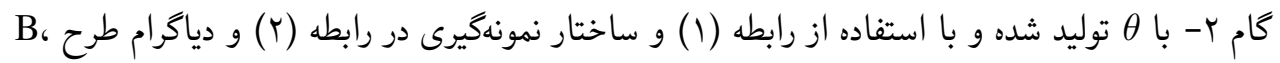

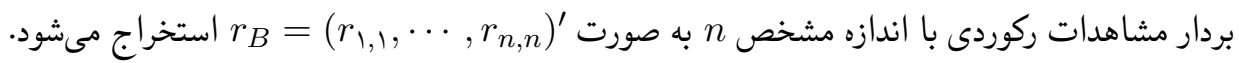

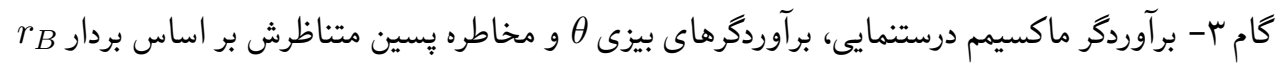

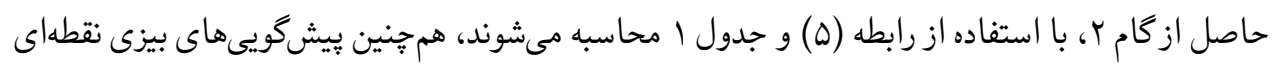

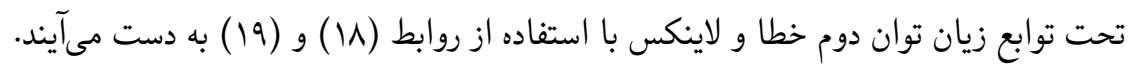

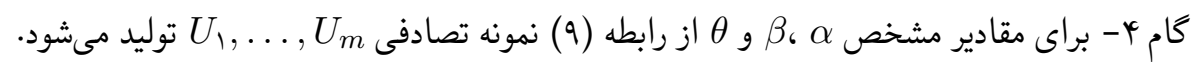

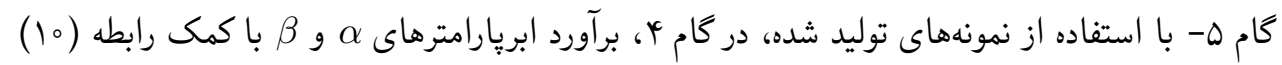

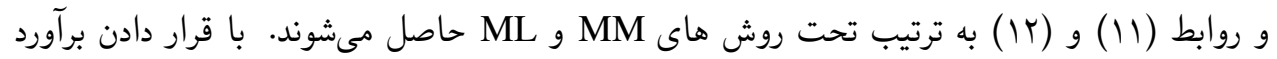

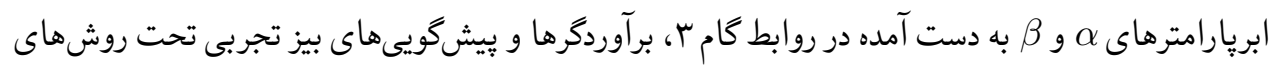
MM و ML كامو- عبارتهاى

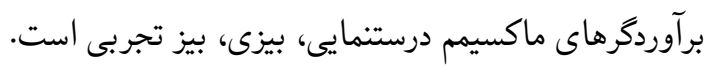

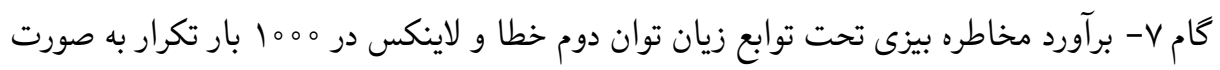

$$
E R_{\uparrow}\left(\hat{\theta}_{i}\right)=\frac{1}{1000} \sum_{i=1}^{1000} L_{\curlywedge}\left(\theta, \hat{\theta}_{i}\right), \quad E R_{\Upsilon}\left(\hat{\theta}_{i}\right)=\frac{1}{1000} \sum_{i=1}^{1000} L_{\Upsilon}\left(\theta, \hat{\theta}_{i}\right)
$$

محاسبه مىشوند. مقادير كوجكتر اين معيار حاكى از دقت بيشتر و عملكرد بهتر است. كام

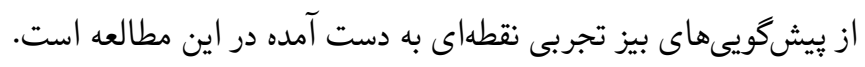

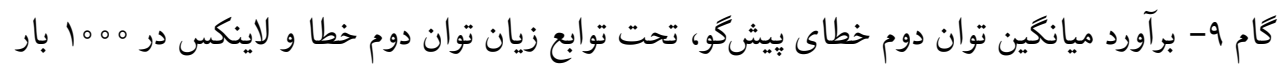
تكرار به صورت زير محاسبه مىشوند.

$$
\operatorname{MSPE} E_{\curlywedge}\left(\hat{Y}_{i}, Y_{i}\right)=\frac{1}{1000} \sum_{i=1}^{1000} L_{\curlywedge}\left(Y_{i}, \hat{Y}_{i}\right), \operatorname{MSPE} E_{\curlyvee}\left(\hat{Y}_{i}, Y_{i}\right)=\frac{1}{1000} \sum_{i=1}^{1000} L_{\uparrow}\left(Y_{i}, \hat{Y}_{i}\right)
$$


كام 1 - مقادير احتمال يوشش CP و ميانخين طول بازه EL ، در 000 بار تكرار به صورت

$$
E L=\frac{1}{1000} \sum_{r=1}^{1000}\left(L_{r}-U_{r}\right), \quad C P=\frac{1}{1000} \sum_{r=1}^{1000} I\left(L_{r} \leq Y_{i} \leq U_{r}\right)
$$

محاسبه مىشوند، كه در آن II I تابع نشانگر مجموعه A است. جدول ץ برآوردگرهاى بيزى و ماكسيمم

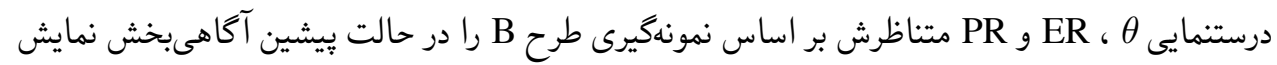

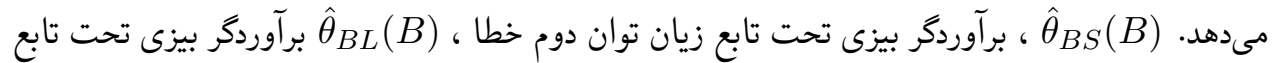

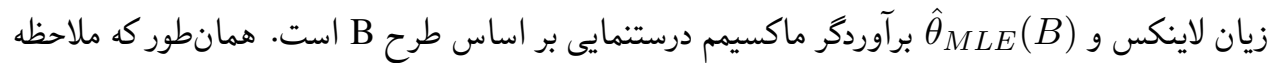

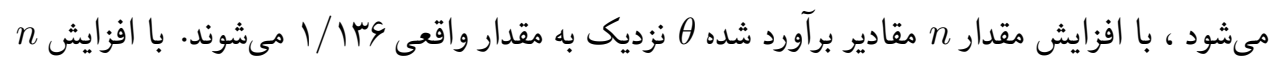

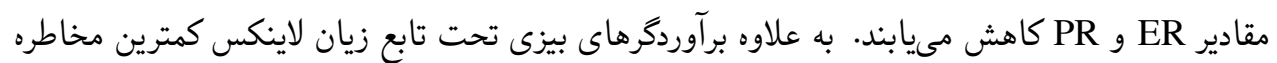

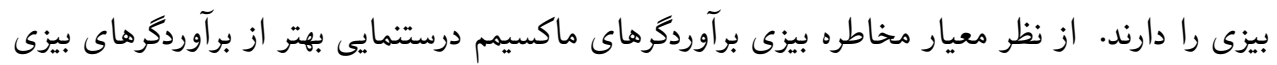

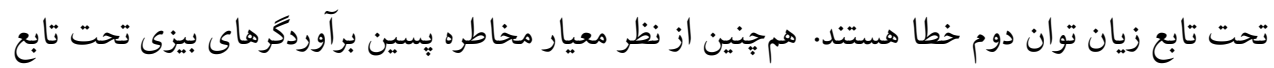

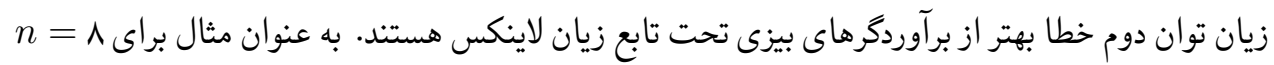
و ه

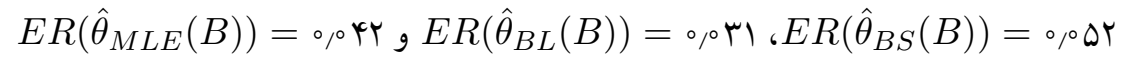

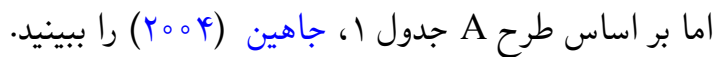

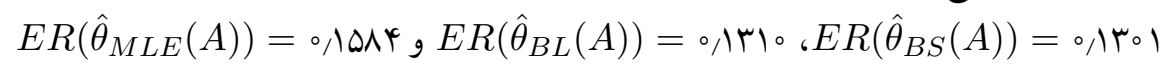

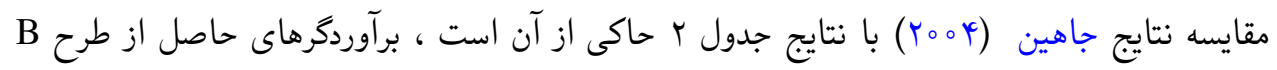

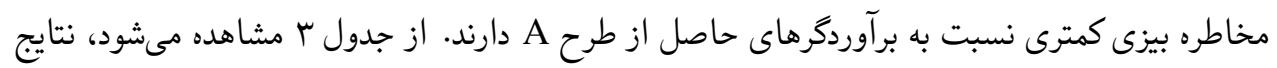

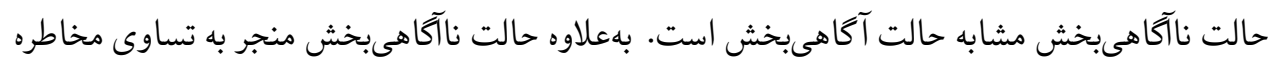
بيزى برآوردكر ماكسيمم درستنمايى و برآوردكر بيزى تحت تابع زيان توان دوم خطا شان شده است.

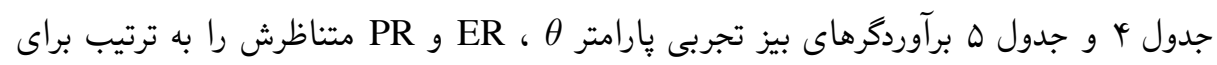

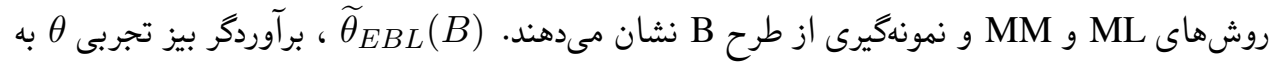

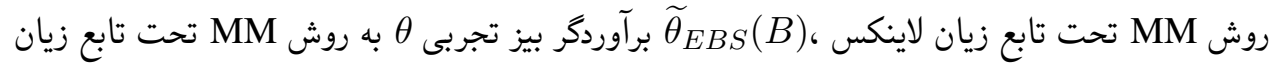
توان دوم خطا ، (B)

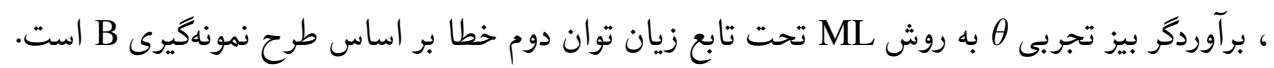

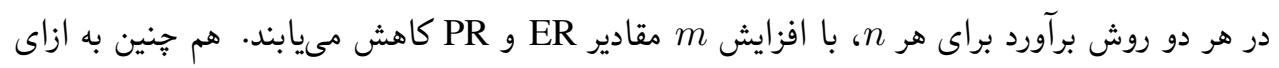


مقايسه برآوردها و ويشكويىهاى بيز تجربى

جدول r. برآوردكرهاى بيزى و ماكسيم درستنمايى ، ER و PR متناظرش بر اساس نمونهيرى از طرح B در حالت آكاهىبخش

\begin{tabular}{|c|c|c|c|c|c|c|}
\hline \multicolumn{6}{|c|}{ LINEX } & \multirow[b]{3}{*}{$n$} \\
\hline \multicolumn{3}{|c|}{$a=10$} & \multicolumn{3}{|c|}{$a=0$} & \\
\hline$E R\left(\hat{\theta}_{B L}(B)\right)$ & $P R\left(\hat{\theta}_{B L}(B)\right)$ & $\hat{\theta}_{B L}(B)$ & $E R\left(\hat{\theta}_{B L}(B)\right)$ & $P R\left(\hat{\theta}_{B L}(B)\right)$ & $\hat{\theta}_{B L}(B)$ & \\
\hline$\circ / \circ \wedge^{\circ}$ & $V / T M I$ & $0 / 94 \Lambda$ &.$/ 091$ & $r / 490$ & $1 / 199$ & $r$ \\
\hline$\% \Delta \Delta r$ & $r / \Delta+4$ & $1 / 014$ & .094 & $1 / 0.4 \pi$ & $1 / 109$ & $\Delta$ \\
\hline$\% \%$ Y & $1 / 9 \pi r$ & $1 / 0 v 1$ & $\%$ &.$/$ KAV & $1 / 140$ & $\wedge$ \\
\hline
\end{tabular}

\begin{tabular}{|c|c|c|c|c|c|}
\hline \multicolumn{2}{|c|}{ MLE } & \multicolumn{3}{|c|}{ SEL } & \\
\hline$E R\left(\hat{\theta}_{M L E}(B)\right)$ & $\hat{\theta}_{M L E}(B)$ & $E R\left(\hat{\theta}_{B S}(B)\right)$ & $P R\left(\hat{\theta}_{B S}(B)\right)$ & $\hat{\theta}_{B S}(B)$ & $n$ \\
\hline$\circ / 9 \circ \Delta$ & $1 / 441$ & $0 / 941$ & o/rYq & $1 / 991$ & $r$ \\
\hline o/TrV & $1 / Y 19$ &.$/ I V Y$ &.$/ 101$ & $1 / 499$ & 0 \\
\hline$\%$ ort & $1 / 199$ & $\% \Delta \Delta r$ & $\%$ & $1 / r \mu 4$ & $\wedge$ \\
\hline
\end{tabular}

جدول ب. برآوردخرهاى بيزى و ماكسيم درستنمايى 0 ، PR و ER متناظرش بر اساس نمونهكيرى از طرح B در حالت

\begin{tabular}{|c|c|c|c|c|c|c|}
\hline \multicolumn{6}{|c|}{ LINEX } & \multirow[b]{3}{*}{$n$} \\
\hline \multicolumn{3}{|c|}{$a=10$} & \multicolumn{3}{|c|}{$a=\Delta$} & \\
\hline$E R\left(\hat{\theta}_{B L}(B)\right)$ & $P R\left(\hat{\theta}_{B L}(B)\right)$ & $\hat{\theta}_{B L}(B)$ & $E R\left(\hat{\theta}_{B L}(B)\right)$ & $P R\left(\hat{\theta}_{B L}(B)\right)$ & $\hat{\theta}_{B L}(B)$ & \\
\hline$. / r+q$ & $9 / \Lambda_{0}$ &.$/ 911$ & $. / 1+4$ & $r / A T V$ & $\circ / \wedge V Y$ & $r$ \\
\hline .091 & $r /$ rAN & -/AVq & $\% \circ 1$ & $1 / 0 Y Y Y^{\prime}$ & $1 / 011$ & 0 \\
\hline \multirow[t]{6}{*}{$\% / r q$} & $1 / 091$ & $1 / \circ v$ & r &.$/$ KrV & $1 / \circ \vee \wedge$ & $\wedge$ \\
\hline & \multicolumn{2}{|c|}{ MLE } & \multicolumn{3}{|c|}{ SEL } & \\
\hline & $E R\left(\hat{\theta}_{M L E}(B)\right)$ & $\hat{\theta}_{M L E}(B)$ & $E R\left(\hat{\theta}_{B S}(B)\right)$ & $P R\left(\hat{\theta}_{B S}(B)\right)$ & $\hat{\theta}_{B S}(B)$ & $n$ \\
\hline & $\circ / 9 \circ D$ & $1 /$ r41 &.$/ 400$ &.$/ r_{0} 1$ & $1 /$ ral & $r$ \\
\hline &.$/ K Y$ & $1 / Y 19$ &.$/ T Y$ &.$/ 10 \mathrm{~V}$ & $1 / Y 19$ & $\Delta$ \\
\hline & $\%$ \% Y & $1 / 199$ & \%OKY & $\% \%$ & $1 / 199$ & $\wedge$ \\
\hline
\end{tabular}

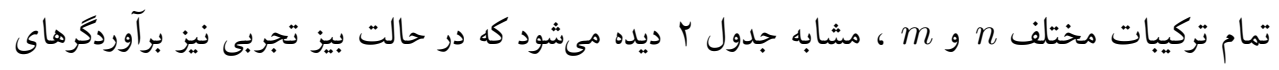

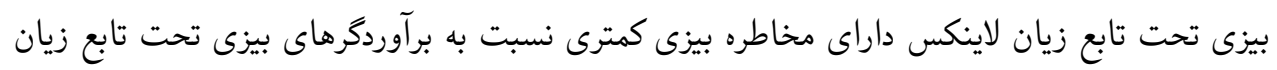

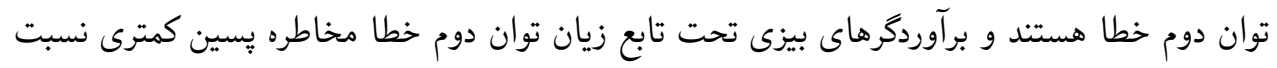

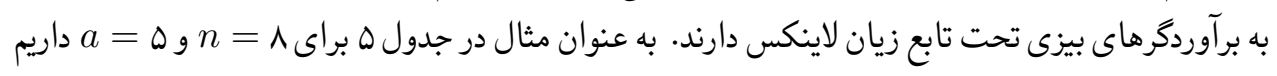

$$
E R\left(\widetilde{\theta}_{E B S}(B)\right)=\left\{\begin{array}{ll}
0,0 \Delta r & \mathrm{~m}=10 \\
0,0 \mathrm{rV} & \mathrm{m}=r_{0}
\end{array} \quad E R\left(\widetilde{\theta}_{E B L}(B)\right)= \begin{cases}0,0 \mu r & \mathrm{~m}=10 \\
0,0 \mathrm{rq} & \mathrm{m}=r_{0}\end{cases}\right.
$$

اما بر اساس طرح A (جدول ا ، جاهين (Y.Y (Y) را ببينيد): 


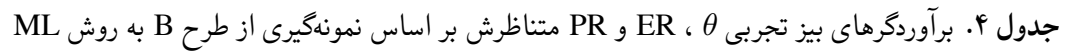

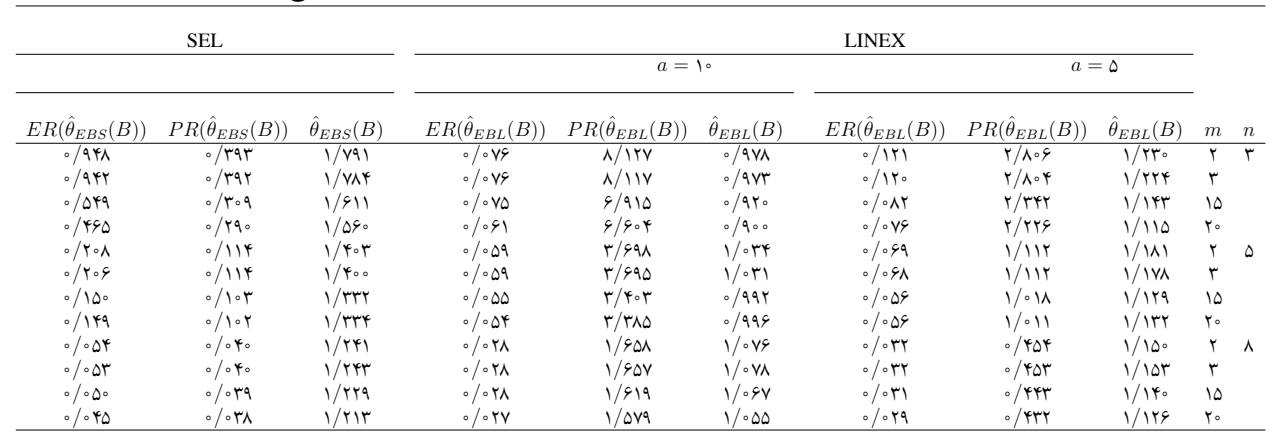

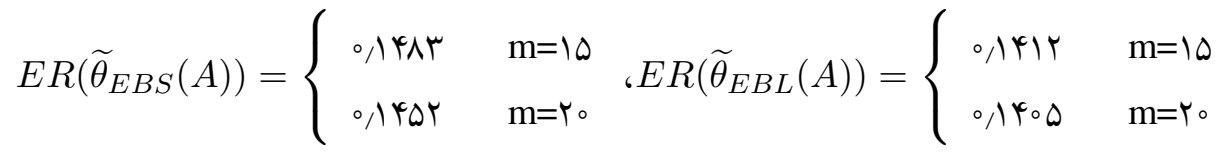

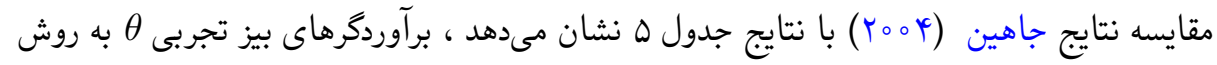
B حسبت به برآوردكرهاى بيز تجربى حاصل از طرح A A مخاطره بيزى كمترى دارند.

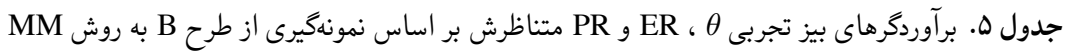

\begin{tabular}{|c|c|c|c|c|c|c|c|c|c|c|}
\hline \multicolumn{3}{|c|}{ SEL } & \multicolumn{6}{|c|}{ LINEX } & \multirow[b]{3}{*}{$m$} & \multirow[b]{3}{*}{$n$} \\
\hline & & & \multicolumn{3}{|c|}{$a=1$ 。 } & \multicolumn{3}{|c|}{$a=\Delta$} & & \\
\hline$E R\left(\widetilde{\theta}_{E B S}(B)\right)$ & $\operatorname{PR}\left(\widetilde{\theta}_{E B S}(B)\right)$ & $\widetilde{\theta}_{E B S}(B)$ & $E R\left(\widetilde{\theta}_{E B L}(B)\right)$ & $\operatorname{PR}\left(\widetilde{\theta}_{E B L}(B)\right)$ & $\widetilde{\theta}_{E B L}(B)$ & $E R\left(\widetilde{\theta}_{E B L}(B)\right)$ & $\operatorname{PR}\left(\widetilde{\theta}_{E B L}(B)\right)$ & $\widetilde{\theta}_{E B L}(B)$ & & \\
\hline$r / \Delta r t$ & \% IAY & $r / 9 A V$ & \%/MT & $9 /$ TSY & $Y / \circ 0_{0}^{\circ}$ & $1 / \% O Y$ & $1 /$ AVI & $r / Y I Y$ & 10 & $r$ \\
\hline $0 / T K Y$ & $0 / 1+9$ & $1 / 949$ & $\% \Delta \Delta$ & $r / 9 \Delta r$ & $1 / 1 A^{4}$ & $\%$ \% & $1 / * \mid T$ & $1 / 499$ & $r_{0}$ & \\
\hline $0 / 490$ & $0 / 11 \%$ & $1 / 499$ & $\% \% \psi r$ & $r / v y_{0}$ & $1 / N T$ & $\% \propto \mu$ & 1/Kro & $1 /$ rVt & 10 & 0 \\
\hline$\% / T \Lambda$ & $\% \vee \gamma \wedge$ & $1 / T M$ & $\%$ TV & $r / A \varphi Y$ & $1 / 104$ & $\% * 4$ & $\% / A Y_{0}$ & $1 / K Y 4$ & ro & \\
\hline$\% \Delta r$ & $\% \psi_{0}$ & $1 / T r 4$ & $\%$ \% & $1 / 9 \psi_{0}$ & $1 / \circ v$ & $\% \pi r$ & $0 / 4+9$ & $1 / 14 \Delta$ & 10 & $\wedge$ \\
\hline$\% \% \mathrm{Fv}$ & $\%$ \% $\lambda$ & $1 / K T T$ & $\% \%+\Lambda$ & $1 / \Delta \wedge r$ & $1 / .94$ & $\% \%+9$ & $0 / 4 \pi r$ & $1 / 149$ & ro & \\
\hline
\end{tabular}

جدول 9 مقادير بيشگيى بيز تجربى براى sامين ركورد آينده و MSPE متناظرش تحت توابع زيان

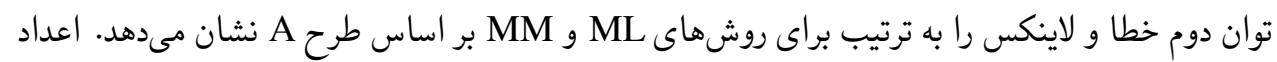

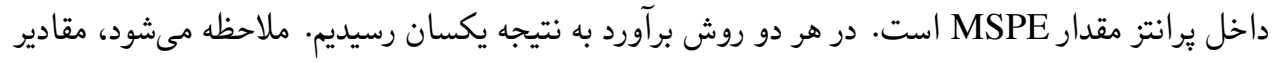

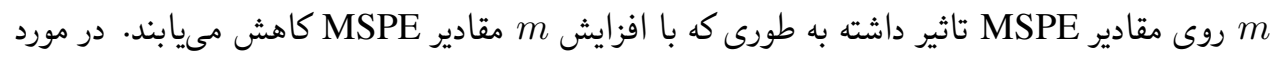

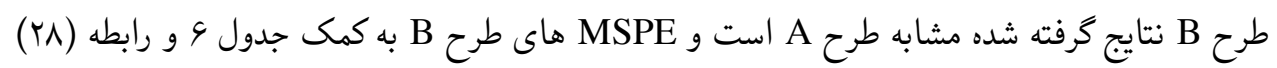
قابل استخراج است.

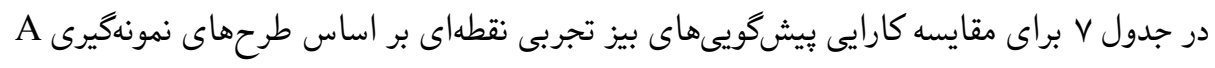


مقايسه برآوردها و ييشكويىهاى بيز تجربى . .

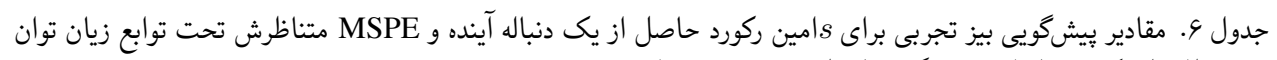

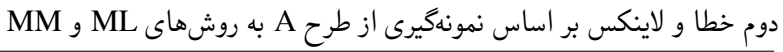

\begin{tabular}{|c|c|c|c|c|c|c|c|c|}
\hline \multicolumn{2}{|c|}{ SEL } & \multicolumn{4}{|c|}{ LINEX } & \multirow[b]{3}{*}{$m$} & \multirow[b]{3}{*}{$n$} & \multirow[b]{3}{*}{ 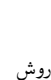 } \\
\hline & & \multicolumn{2}{|c|}{$a=10$} & \multicolumn{2}{|c|}{$a=\Delta$} & & & \\
\hline$s=11$ & $s=9$ & $s=11$ & $s=9$ & $s=11$ & $s=9$ & & & \\
\hline$\Lambda / I \circ r(|V /| r \mid)$ & q/VOF (II/৭৯।) & $r / 9 V Y(r \Delta / \wedge \Delta I)$ & $r / 9 \Lambda 1\left(r_{\circ} / q \Delta r\right)$ & $\psi / \Delta \circ Y(T \circ / \xi \circ Y)$ & $4 / 100(1 N / 011)$ & $r$ & $r$ & ML \\
\hline$\Lambda / r \circ r(\mid g / 9 V I)$ & $\left.9 / \operatorname{Arr}(11 / \mathrm{V})_{0}\right)$ & $r / q \wedge r(r \Delta / q V i)$ & $r / 9 q_{0}\left(r_{0} / \Lambda K r\right)$ & $r / \Delta r r\left(r_{0} / r T H C\right)$ & $\psi / I T I(I V / \Lambda \Delta T)$ & r & & \\
\hline $1 / r 91(19 / 09 Y)$ & $G / \Lambda G Y(11 / G V Y)$ & $r / 99 \circ(r \Delta / \varphi \circ r)$ & $r / \gamma_{0} r\left(r_{0} / \Lambda l_{0}\right)$ & $r / \Delta r I(r \circ / r r I)$ & $r / \operatorname{Irr}(\mathrm{IV} / \mathrm{Vqr})$ & 10 & & \\
\hline$\Lambda / T r t(19 / T \mid Y)$ & $G / 9 r r(11 /$ ror $)$ & $\psi / 001(r \Delta / Y \Delta T)$ & $r / V I r\left(r_{0} / V r r\right)$ & $r / \Delta \Delta r(r \% / 0 \mid r)$ & $4 / \mathrm{Kr}(\mathrm{IV} / G G \mathrm{~T})$ & ro & & \\
\hline$\Lambda / A \|(I r / \Delta \Delta r)$ & $V / r \Delta r(q / r+1)$ & $\Delta / \Delta \circ r(r I / q T I)$ & $0 / \operatorname{IVT}(\mathrm{II} / A T r)$ & $\Delta / q V_{0}(\mid N / V+r)$ & $\Delta / * q 1(10 / * \Delta *)$ & r & 0 & \\
\hline N/ & $V / \psi_{0} \mid(q / \mid \wedge \psi)$ & $0 / \Delta \|\left(K I / \wedge r_{0}\right)$ & $0 /$ IVO $(11 /$ VAr $)$ & $0 / 991(1 K / 9 \circ Y)$ & $0 / 0.4(10 / 411)$ & r & & \\
\hline$\Lambda / \wedge q I\left(\mathrm{Kr} / \Delta \mathrm{Ar}^{\prime}\right)$ & $v / 4 \circ r(q / 191)$ & O/DIT(YI/VAr) & $0 /|V|\left(11 / V_{\Delta I}\right)$ & $\Delta / \wedge q T(I N / \Delta \Delta Y)$ & $\Delta / \Delta . r(10 / T \Delta r)$ & 10 & & \\
\hline$\Lambda / 9 Q_{0}(\mathrm{Ir} / \Lambda \subseteq Y)$ & $V / \operatorname{RVY}(q / 194)$ & O/OTY(YI/VOT) & $0 / 111(11 / v+1)$ & $4 / 001(1 \mathrm{~N} / 4 G 4)$ & $\Delta / \Delta T I(10 / T Y)$ & ro & & \\
\hline $9 / / \Lambda \cdot(10 / 191)$ & $V / \Lambda r r(V / r r Y H)$ & $V / 991(10 / \Delta \Lambda r)$ & $V / T V F\left(q / q V_{0}\right)$ & V/arr (a/AGr) & $V / r 4 \circ(9 / 901)$ & r & $\wedge$ & \\
\hline$q /$ rq1 (10/10r) & 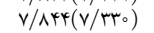 & $V / S V T(10 / \Delta G Y)$ & $V / Y V I(G / G G Y)$ & $\mathrm{V} / \mathrm{qu}\left(\mathrm{q} / \mathrm{\Lambda} \mathrm{F}_{0}\right)$ & $V /$ MGY (G/GOH) & $r$ & & \\
\hline$q / 4 \circ r(10 / 11 \%)$ & $V / \lambda F I(V / r r r)$ & $V / 9 V Y\left(10 / 0 \%_{0}\right)$ & $V / T V Y(G / 9 \pi r)$ & $V / q \psi_{0}(q / \lambda r I)$ & $V / r G H(G / G \psi Y G)$ & 10 & & \\
\hline$q / \operatorname{tat}(10 / 100)$ & $V / \Lambda G Y(V / T Y Y)$ & $V / G V I(10 / 0 F Y)$ & $V / T V Y\left(G / G Y_{0}\right)$ & $V / q \Delta r(q / \Lambda \circ r)$ & $v / r v_{0}(q / q 4 i)$ & ro & & \\
\hline$q / r \varphi_{0}(r I / r r I)$ & $V / 9 \circ r(1 \Delta / \circ \Delta \psi)$ & $r / 001(r \Delta / q \mid r)$ & $r / r \mid r\left(r_{0} / V \Lambda_{0}\right)$ & $r / \operatorname{DST}\left(\mu_{0} / \mid K T\right)$ & $r / \mid \varphi_{0}(|V / V Y|)$ & 10 & $r$ & $\mathrm{MM}$ \\
\hline $1 / 9 r T(19 / T r T)$ & $v / r V_{0}(|r / \Delta r|)$ & $r / \circ r(r \Delta / \Delta r \psi)$ & $r / V I I\left(r_{0} / V q r\right)$ & $\psi / \Delta G \psi\left(\mu_{0} / \Delta_{0}\right)$ & $r / 19 r(1 V / 9 V H)$ & $r_{0}$ & & \\
\hline $9 / \operatorname{\Delta rt}\left(10 / 410^{\circ}\right)$ & $V / \operatorname{Arr}(10 / 9 \Delta r)$ & $\Delta / \Delta \psi_{0}(Y I / V Y I)$ & $\Delta / 19 T(11 / V Y Y)$ & $G / \circ \psi 1(K / H \circ \psi r)$ & $\Delta / \Delta \mu \psi\left(10 / \mu T_{0}\right)$ & 10 & 0 & \\
\hline$q / r \Delta D(\mid k / \Delta . r)$ & $V / V \circ 4\left(10 / 1 r_{0}\right)$ & $0 / \Delta T_{T}\left(r_{0} / V T r\right)$ & $0 / 190(10 / V+1)$ & $4 / 0 r r(I V / K r Y)$ & $0 / \Delta H I(q /$ KKY $)$ & ro & & \\
\hline$q / \Delta q r(10 / V H Y)$ & $V / 901(V / \Delta . r)$ & $\mathrm{V} / 9 \mathrm{~N}+\left(10 / 0 \mathrm{H}_{0}\right)$ & V/rAT $(8 / 9 G T)$ & $V / 990\left(9 / \Lambda_{0} 1\right)^{\prime}$ & $V / r V r(\varphi / 9 \Delta t)$ & 10 & $\wedge$ & \\
\hline $9 / \operatorname{art}(10 / 010)$ & 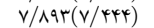 & $V / 911(10 / K Y Y)$ & $V / T V \psi\left(\varphi / 4 \varphi_{0}\right)$ & $V / 9 \Delta r(9 / 9 K T)$ & $V / r V_{0}(\varphi / \mu \psi+1)$ & ro & & \\
\hline
\end{tabular}

و B، مقادير معيار RE با استفاده از رابطه (YN) تحت توابع زيان لاينكس و توان دوم خطا به روشهاى

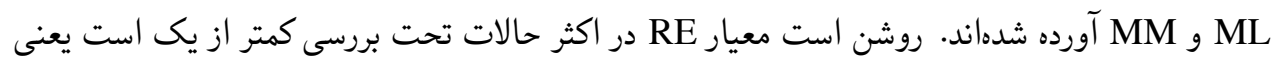

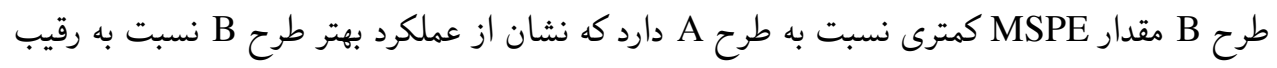

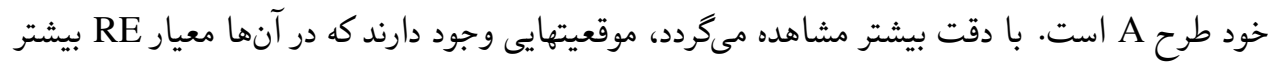

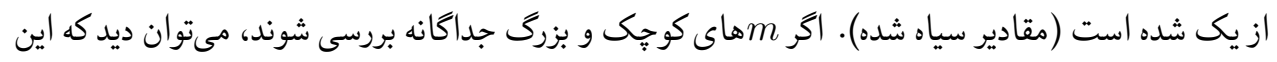

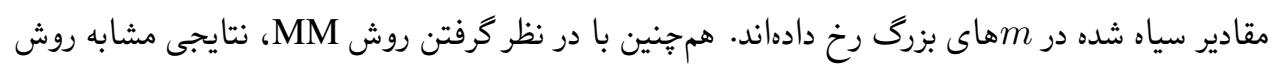

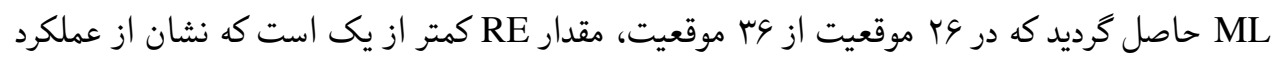

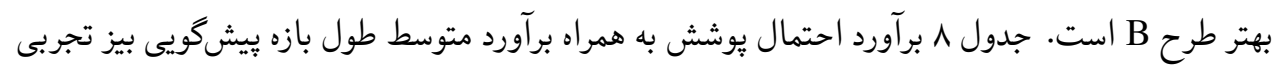

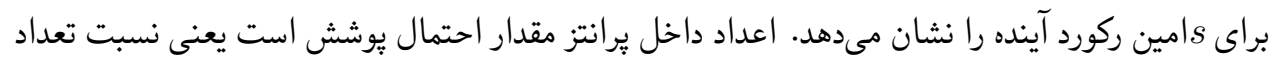

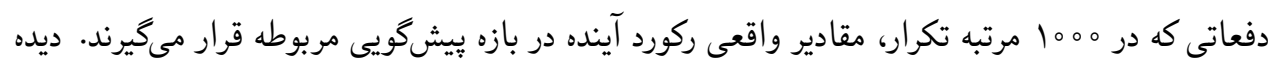

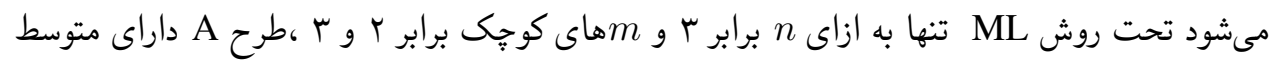

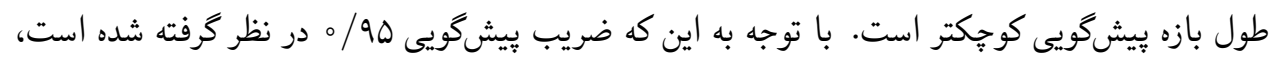

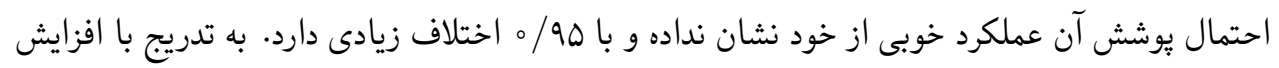

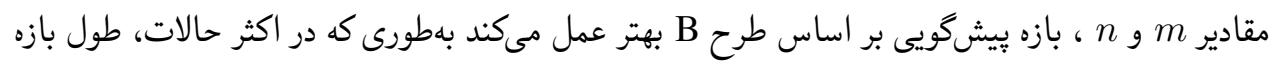

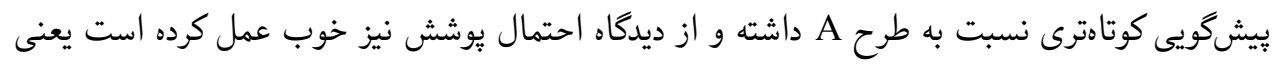




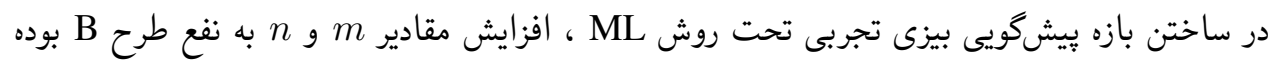

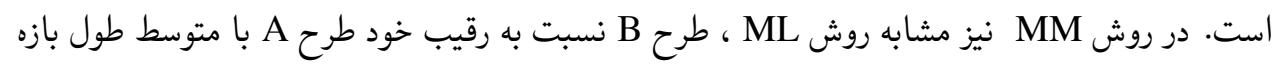
ييشگويى كوتاهتر و احتمال بوشش بهتر ظاهر شده است.

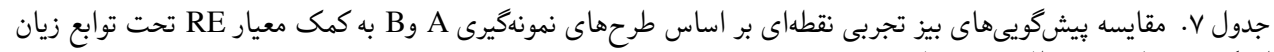

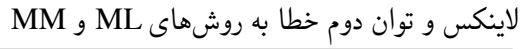

\begin{tabular}{|c|c|c|c|c|c|c|c|c|}
\hline \multicolumn{2}{|c|}{ SEL } & \multicolumn{4}{|c|}{ LINEX } & \multirow[b]{3}{*}{$m$} & \multirow[b]{3}{*}{$n$} & \multirow[b]{3}{*}{ روش } \\
\hline & & \multicolumn{2}{|c|}{$a=1 \circ$} & \multicolumn{2}{|c|}{$a=\Delta$} & & & \\
\hline$s=11$ & $s=9$ & $s=11$ & $s=9$ & $s=11$ & $s=9$ & & & \\
\hline $0 / 94 \Delta$ &.$/ 9 \Delta \Delta$ & $0 / 949$ & $0 / 9 \Delta \psi$ & $0 / 94 \lambda$ & $0 / 914$ & $r$ & $r$ & ML \\
\hline.$/ 910$ & $\circ / \wedge 91$ & -/GTF & - /GTF &.$/ 948$ & سד/94. & $r$ & & \\
\hline $0 / 90 r$ & $\circ / \Lambda \Lambda 1$ &.$/ 910$ & -/arr &.$/ 9 \mu r$ &.$/ 941$ & 10 & & \\
\hline - / $\wedge$ ro & -/ATV & - /MAr & $0 / 904$ & $0 / 919$ & - / $\Delta \Delta \Delta$ & ro & & \\
\hline $0 / 949$ & ०/ qvq &.$/ 990$ & $0 / 9 \Lambda 4$ & $0 / 9 G 4$ &.$/ 990$ & $r$ & 0 & \\
\hline $0 / 904$ &.$/ q \wedge \mu$ &.$/ 919$ &.$/ 9 \wedge F$ & $0 / 9 \gamma_{0}$ &.$/ 9 \vee \wedge$ & $r$ & & \\
\hline$\circ / \wedge 91$ & $0 / q \mu y$ &.$/ 919$ &.$/ 9 \vee \wedge$ &.$/ 94_{0}$ & $1 / \circ r \Lambda$ & 10 & & \\
\hline$\circ / \Lambda \Delta^{\circ}$ & $\circ / 901$ &.$/ 911$ &.$/ 99 V$ &.$/ 949$ & $1 / \circ r^{4}$ & ro & & \\
\hline$\circ / 9 V_{0}$ & $0 / 919$ & $\circ / 9 \vee q$ & $0 / 994$ &.$/ 9 \vee 1$ &.$/ 991$ & r & $\wedge$ & \\
\hline.$/ 949$ & - $/ 9 \wedge \Delta$ &.$/ 949$ &.$/ 910$ &.$/ 900$ &.$/ 991$ & $r$ & & \\
\hline $0 / 9 V 1$ & $0 / 994$ & $1 / \circ \Delta \Lambda$ & $0 / 9 V^{4}$ & $1 / 040$ &.$/ 991$ & 10 & & \\
\hline.$/ 901$ & $\circ / 990$ & $1 / 009$ & $1 / 011$ & $1 / \circ r V$ & $1 / 00 Y$ & ro & & \\
\hline $0 / 911$ & ./Vkt & $\circ / q \gamma_{0}$ &.$/ 911$ & - /qrם & $0 / 990$ & 10 & $r$ & MM \\
\hline ./VTr & $\circ / v 90$ & $\circ / 9 \mu_{0}$ & $\circ / 9 \circ \Delta$ & س &.$/ 91 r$ & ro & & \\
\hline -/AVr &.$/ 994$ & $\circ / \wedge \Delta_{0}$ & $\circ / \wedge \Lambda 1$ &.$/ q \& V$ & $0 / 904$ & 10 & $\Delta$ & \\
\hline 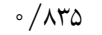 &.$/ 9 M$ & - $/ M \Lambda \Lambda$ & -/ADT & $0 / 991$ & - / ४४ & ro & & \\
\hline.$/ 99 V$ & $1 / K \mid{ }^{4}$ & I/TOG & $1 / 1 \% 4$ & $1 / 0 \wedge 9$ & $1 / \mu_{0}$ & 10 & $\wedge$ & \\
\hline $1 / 01 Y$ & $1 / 1 \% V$ & $1 / 100$ &.$/ 9 \wedge \Lambda$ & $1 / Y 10$ & $1 /$ roo & ro & & \\
\hline
\end{tabular}

\section{V مثال كاربردى V}

در اين بخش، مباحث مطرح شده در بخشهاى قبل روى يك مجموعه داده واقعى به كار كرفته مىشود.

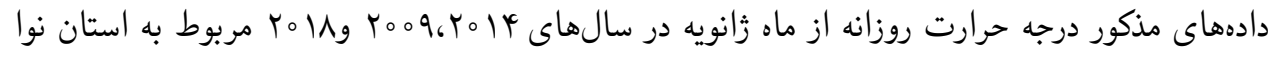

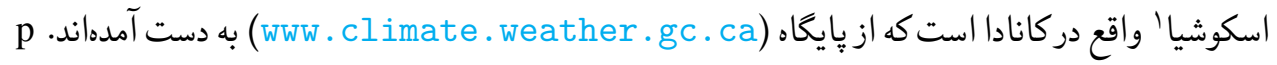

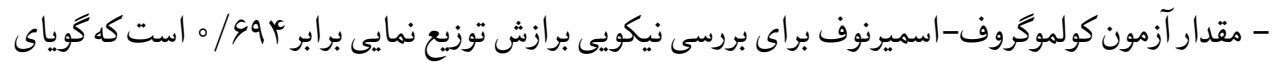
مناسب بودن توزيع نمايى روى دادهها است. بردارهاى $r_{A}=0$ )

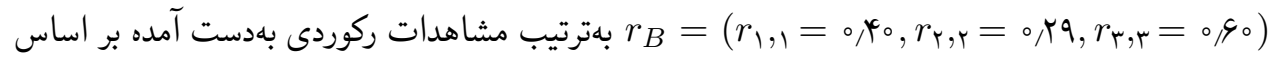


F

جدول ^. متوسط احتمال يوشش و طول بازهاى بيش وقويى بيز تجربى براى sامين ركورد دنباله آينده بر اساس طرحهاى A و

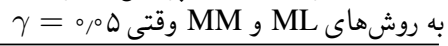

\begin{tabular}{|c|c|c|c|c|c|c|}
\hline \multicolumn{2}{|c|}{ Bر نهونهيرى } & \multicolumn{2}{|c|}{ طرح نمونهكيرى } & \multirow[b]{2}{*}{$m$} & \multirow[b]{2}{*}{$n$} & \multirow[b]{2}{*}{ 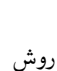 } \\
\hline$s=11$ & $s=9$ & $s=11$ & $s=9$ & & & \\
\hline$(\circ / V Y I) \mid r / \Lambda \circ Y$ & $\left.\left(\circ / \varphi^{*}\right)\right) 11 / \Lambda_{0 \circ}$ & $(0 / V Y T) \| Y / 9 V T$ & $\left.\left(\circ / V Y^{\circ}\right)\right) 10 / V \Delta 1$ & r & $r$ & ML \\
\hline$(0 / M Y Y) \mid r / \Delta V_{0}$ & $(0 / 9 \circ \%) \| 11 / 914$ & $\left(0 / Y_{0}\right) \mid r / 901$ & $\left(0 / V_{k r}\right) 10 / V_{k+\alpha}$ & r & & \\
\hline 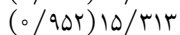 & $(0 / 900) \mid r / 1.01$ & $(0 / 9 \pi r) \mid 9 /$ Kry & $(0 / q K I) \mid r / q K r$ & 10 & & \\
\hline$(0 / 900) 10 / \% 01$ & $(0 / 9 \Delta r) \mid r / I r r$ & $(0 / 941) 19 / 914$ & $(0 / Q Q Y Y) \mid Y / O V_{0}$ & ro & & \\
\hline$(0 / q V r) \mid r / r q Y$ & $(0 / 9 \Delta 1) 11 / \Delta V Y$ & $(0 / \Lambda G Y) \mid r / \Delta \Delta^{\circ}$ & $(0 / \wedge V T) \| / \Delta G Y$ & r & 0 & \\
\hline$(0 / 971) 1 r / 194$ & $\left.(0 / q \wedge)^{*}\right) 11 / 4 q 0$ & (0/AKr)Ir/rrT & $\left(0 / \wedge \varphi_{0}\right) \| /{ }^{\prime} \Lambda I$ & r & & \\
\hline$(0 / 994) \mid r / 940$ & $(0 / 99 T) I T / 04 T$ & $(0 / 900) 10 / V 91$ & $(0 / q \Delta r)$ Ir/ / & 10 & & \\
\hline (0/9Ar)Ir/9Ar & $(0 / 990) 11 / 941$ & $(0 / 9 G \%) \backslash G /$ MA & $(0 / 991) \mid r / \Lambda \circ r$ & $r_{0}$ & & \\
\hline$(0 / 990) \mid r / r q 1$ & $(0 / 994) 10 / 994$ & $(0 / 911) 1 r / V H r$ & $(0 / 9 Y Y)) 11 / 110$ & r & $\wedge$ & \\
\hline$(0 / 99 \%) \mid r / K_{0} Y$ & $(0 / 991) 10 / 9 V Y$ & $(0 / 91 \%) \mid r / 900$ & $(0 / 9 T r) \| / V_{0} r$ & r & & \\
\hline$(0 / 991) \| r / 90 \%$ & $(0 / 994) \| 1 / 140$ & $(0 / 9 V Y) \mid Y / \Delta V r$ & $\left(0 / 9 V_{0}\right) \mid r / \Lambda_{0} 1$ & 10 & & \\
\hline$(0 / 994) \| r / V_{0} \circ$ & $(0 / 99 Y) 11 /$ TYT & $\left(0 / 9 V_{0}\right) \mid 0 / T+1$ & $(0 / 9 \Lambda \mu) \mid r / 104$ & ro & & \\
\hline$(0 / 944) 10 / 194$ & $(0 / q \Delta \psi) \mid r / 00$ & $(0 / q Y r) Y I / V V^{F}$ & $(0 / 901) M / K I T$ & 10 & $r$ & MM \\
\hline$(0 / 9 \Delta \psi) \mid r / q 10$ & $(0 / 9 \Delta r) \mid r / 9 q r$ & $(0 / 900) 19 / 211$ & $(0 / 90 \mathrm{~T}) \mathrm{lq} / \mathrm{VqT}$ & $r_{0}$ & & \\
\hline$(0 / 99 Y) \mid Y / \circ V T$ & $(0 / 990) \mid r / r \Delta 1$ & $(0 / 9 G Y) 19 / \Delta H Y$ & $(0 / 9 V I) 19 / \Delta V Y$ & 10 & 0 & \\
\hline$\left(0 / 9 \Lambda_{0}\right) \mid r / \Delta 01$ & $(0 / 994) \| / 114$ & $(0 / 991) \backslash N /$ KrT & $\left.(0 / 9 V)^{\circ}\right) 10 / 900$ & ro & & \\
\hline$(0 / 994) \mid r / V Y 4 F$ & $(0 / 991) 11 / T V Y$ & $(0 / 9 \Delta 4) \mid 9 / 940$ & $(0 / q \Lambda T) I F / T V T$ & 10 & $\Lambda$ & \\
\hline$(0 / 991) 1 Y / \Delta 9 Y$ & $(0 / 999) 11 / 110$ & $(0 / 9 \mathrm{VY}) 19 / 00 \mathrm{r}$ & $\left(0 / 9 \Lambda_{0}\right) \| r / V+1$ & ro & & \\
\hline
\end{tabular}

طرح هاى A و Bستند در نتيجه بيان شد مىتوان ديد اين امكان وجود دارد كه واحدهاى طرح B برخلاف واحدهاى طرح A لزوما مرتب

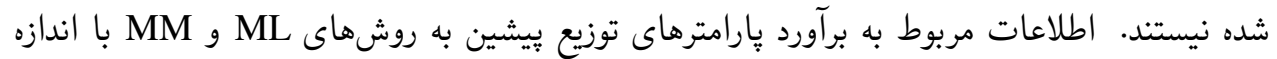

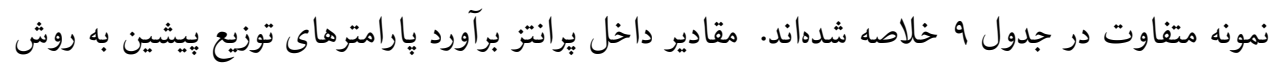

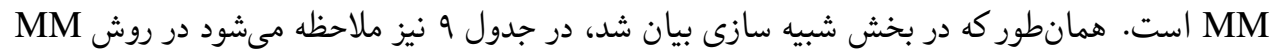

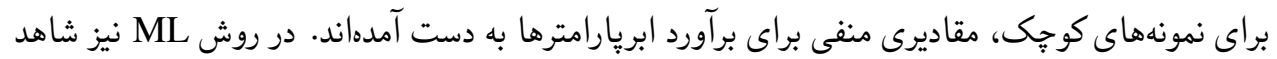

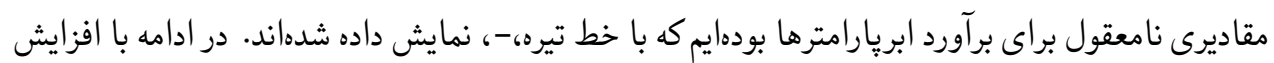

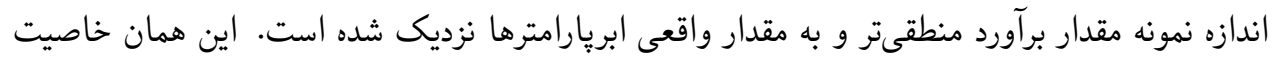
بهينگى مجانبى روش بيز تجربى است كه مطابق انتظار است.

جدول 9 . برآورد ابريارامترها به روشهاى ML و MM براى دادهاى درجه حرارت

\begin{tabular}{|c|c|c|c|c|c|c|c|c|}
\hline \multicolumn{4}{|c|}{ bرح B } & \multicolumn{4}{|c|}{${ }^{A}{ }^{b}$} & \multirow[b]{2}{*}{$m$} \\
\hline$\widetilde{\beta}$ & $\hat{\beta}$ & $\widetilde{\alpha}$ & $\hat{\alpha}$ & $\widetilde{\beta}$ & $\hat{\beta}$ & $\widetilde{\alpha}$ & $\hat{\alpha}$ & \\
\hline$(-1 / K \Psi I)$ & - & $\left(-V / K Y_{0}\right)$ & - & $(-1 / 9 / 4)$ & - & $(-Y / ৭ \circ \Upsilon)$ & - & $r$ \\
\hline$\left(9 / 4 \psi_{0}\right)$ & - & $(r / / \Delta r r)$ & - & $(-1 / K Y Y)$ & $9 / 091$ & $(-Y / Y \circ Y)$ & IF/ATY & $r$ \\
\hline$(r / G Y Y)$ & $\Lambda / \mu I I$ & $\left(\Lambda / \mu \circ r^{\prime}\right.$ & $M / F \Delta F^{\prime}$ & $(0 / 9 K T)$ & $1 / K r$ & $(Y / \circ 11)$ & $r / \Delta . r$ & 10 \\
\hline
\end{tabular}


برخى از نتايج به دست آمده بر اساس طرح هاى A و B به روشهاى ML و MM تحت دادههاى درجه حرارت در جداول زير آمدهاند:

جدول • ا. برآوردكرهاى بيز تجربى $\theta$ و PR متناظرش به روش MM براى دادههاى درجه حرارت

SEL

\begin{tabular}{|c|c|c|c|c|c|c|c|}
\hline \multicolumn{2}{|c|}{ SEL } & \multicolumn{4}{|c|}{ LINEX } & \multirow[b]{3}{*}{$m$} & \multirow[b]{3}{*}{ 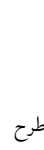 } \\
\hline & & \multicolumn{2}{|c|}{$a=1 。$} & \multicolumn{2}{|c|}{$a=0$} & & \\
\hline$P R\left(\widetilde{\theta}_{B S}(B)\right)$ & $\tilde{\theta}_{B S}(B)$ & $P R\left(\widetilde{\theta}_{B L}(B)\right)$ & $\widetilde{\theta}_{B L}(B)$ & $P R\left(\widetilde{\theta}_{B L}(B)\right)$ & $\tilde{\theta}_{B L}(B)$ & & \\
\hline.$/ 091$ & $r / q Y^{K}$ & IT/KAY & $1 / 09 T$ & $r / \Delta r r$ & $r / \circ r$ & 10 & $\mathrm{~B}$ \\
\hline$r / \Delta \psi 1$ & $r / r 10$ & r./NOT & $1 /|K|$ & IT/VAT & $1 / 901$ & 10 & A \\
\hline
\end{tabular}

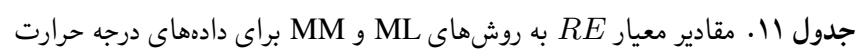

\begin{tabular}{|c|c|c|c|c|c|c|c|}
\hline \multicolumn{2}{|c|}{ SEL } & \multicolumn{4}{|c|}{ LINEX } & \multirow[b]{3}{*}{$m$} & \multirow[b]{3}{*}{ روش } \\
\hline & & \multicolumn{2}{|c|}{$a=1 \circ$} & \multicolumn{2}{|c|}{$a=\Delta$} & & \\
\hline$s=11$ & $s=9$ & $s=11$ & $s=9$ & $s=11$ & $s=9$ & & \\
\hline - Nar & $0, \Lambda \circ \mu$ & $0,94 \Lambda$ & 0,940 & $0, \Lambda \wedge V$ & $0 / \wedge 9 \vee$ & $r$ & ML \\
\hline 0,111 & $0, N \mid \Lambda$ & $0,91 \mathrm{r}$ & 0,941 & $0, \wedge \Lambda 1$ & ० MA & 10 & MM \\
\hline
\end{tabular}

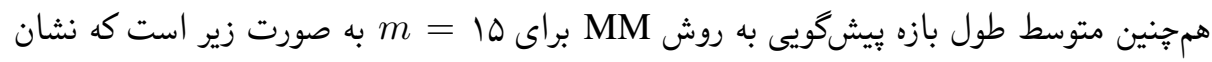
مىدهد مشابه روش ML، طرح BL داراى متوسط طول بازه بيشكويى كوتاهترى نسبت به طرح A است.

$$
E L_{s}(A)=\left\{\begin{array}{ll}
9 / \mathrm{N} / 0 & \mathrm{~s}=9 \\
\mathrm{v} / 911 & \mathrm{~s}=11
\end{array}, \quad E L_{s}(B)= \begin{cases}\Delta / \mathrm{V} / \mathrm{r} & \mathrm{s}=9 \\
4 / 9 \circ \mathrm{r} & \mathrm{s}=11\end{cases}\right.
$$

در اين مثال كاربردى، بار ديخر نتايج حاصل از مطالعه شبيهازى در بخش قبل، مورد تاييد قرار مىگيرد.

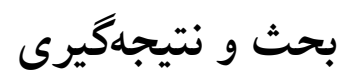

در اين مقاله به موضوع برآورد و يّشكويى در طرحهاى نمونهگيرى مجموعه رتبهدار ركوردى و نمونهيرى

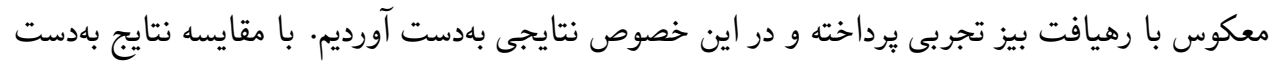


آمده ديده شد، برآوردگرهاى حاصل از شيوه نمونهيرى مجموعه رتبهدار ركوردى كاراتر از برآوردكرهاى حاصل

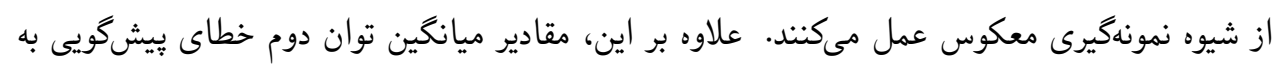

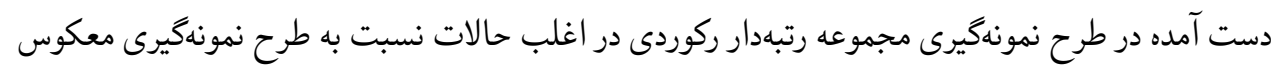

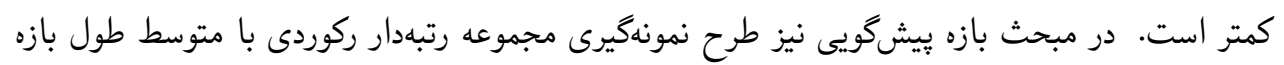

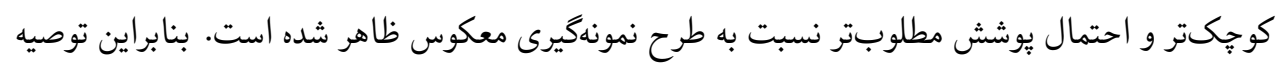

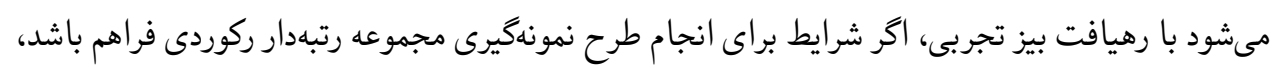
از اين طرح نمونهيرى استفاده شود.

\section{تقدير و تشكر}

نويسندگان مقاله از زحمات سردبير مجله و بيشنهادات ارزنده داوران گرامى و زحمات ويراستار مجله كه

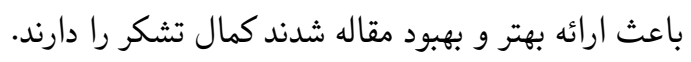

\section{مراجع}

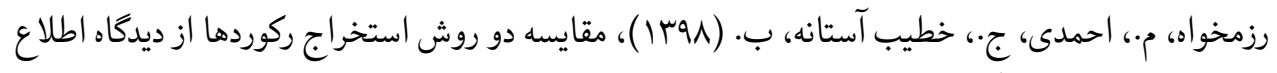

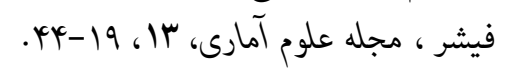

Ahmadi, J. and Balakrishnan, N. (2010), Prediction of Order Statistics and Record Values From Two Independent Sequences, Statistics, 44, 417-430.

Ahsanullah, M. (1995), Record Statistics, Nova Science Publishers Inc, Commack, New York.

Aitchison, J. and Dunsmore, I.R. (1975), Statistical Prediction Analysis, Cambridge University Press, Cambridge.

Arnold, B. C., Balakrishnan, N. and Nagaraja, H.N. (1998), Records, John Wiley \& Sons, New York. 
Carlin, B. P. and Louis, T. A. (2000), Bayes and Empirical Bayes Methods for Data Analysis, 2nd ed. Chapman \& Hall/CRC.

Eskandarzadeh, M., Tahmasebi, S. and Afshari, M. (2016), Information measures for Record ranked set samples, Ciencia e Natura, 38, 554-563.

Geisser, S. (1993), Predictive Inference: An Introduction, Chapman and Hall.

Gulati, S. and Padgett, W.J. (2003), Parametric and Non-Parametric Inference from Record-breaking Data, Lecture Notes In Statistics, 172, Springer, New York.

Jaheen, Z. F. (2004), Empirical Bayes Analysis of Record Statistics Based on Linex and Quadratic Loss Functions, Computers and Mathematics with Applications, 47, 947-954.

Lawless, J. F. (1977), Prediction Intervals for the Two Parameter Exponential Distribution, Technometrics, 19, 469-472.

Maritz, J. L. and Lwin, T. (1989), Empirical Bayes Methods, Section Edition, Chapman and Hall, London.

McIntyer, GA.A. (1952), Method for Unbiased Selective Sampling, Using Ranked Sets, Australian Journal of Agricultural Research, 3, 385-390.

Mirmostafaee, S. M. and Ahmadi, J. (2011), Point Prediction of Future order Statistics From an Exponential Distribution, Statistics and Probability Letters, 81, 360-370.

Nevzorov, V. (2001), Records, Mathematical Theory, Translation of Mathematical Monographs No, 194, American Mathematical Society, Providence, RI. 
Paul, J. and Thomas, P.Y. (2017), Concomitant Record ranked set sampling, Communications in Statistics-Theory and Methods, 46, 9518-9540.

Raqab, M. and Balakrishnan, N. (2008), Prediction Interval for Future Records, Statistics and Probability, 78, 1955-1963.

Safaryian, A., Arashi, M. and Arabi, R. (2019), Improved Estimators for Stress-strength Reliability Using Record Ranked Set Sampling Scheme, Communications in Statistics-Simulation and Computation, https:// doi .org/10 .1080/03610918.2018.1468451.

Salehi, M. and Ahmadi, J. (2014), Record Ranked Set Sampling Scheme, Metron, 72, 351-365.

Salehi, M. and Ahmadi, J. (2015), Estimation of Stress-strength Reliability Using Record Ranked Set Sampling Scheme From the Exponential Distribution, Filomat, 29, 1149-1162.

Salehi, M., Ahmadi, J. and Balakrishnan, N. (2015), Prediction of Order Statistics and Record Values Based on Ordered Ranked Set Sampling, Statistical Computation and Simulation, 85, 77-88.

Salehi, M., Ahmadi, J. and Dey, S. (2016), Comparison of Two Sampling Schemes for Generating Record-breaking Data from the Proportional Hazard Rate Models, Communications in Statistics-Theory and Methods, 45, 3721-3733. 
Journal of Statistical Sciences, Spring and Summer, 2021

Vol. 15, No. 1, pp 193-218

DOI: $10.29252 /$ jss.15.1.193

\title{
Comparison of Empirical Bayesian Estimations and Pre- dictions Based on Record Ranked Set Sampling Scheme with Inverse Sampling Scheme
}

\author{
Golzade Gervi, E. ${ }^{1}$, Nasiri, P. ${ }^{1}$ and Salehi, S. M. ${ }^{2}$ \\ ${ }^{1}$ Department of Statistics, Payame Noor University of Tehran, Tehran, Iran. \\ ${ }^{2}$ Department of Mathematics and Statistics, Neyshabur University, Neyshabur, \\ Iran.
}

\begin{abstract}
The empirical Bayes estimation of the exponential distribution parameter under squared error and LINEX loss functions is investigated when the data collect by the record ranked set sampling scheme method. Then, point and interval predictions for future record values are studied. The results of this sampling scheme are compared with the products of the inverse sampling scheme. To compare the accuracy of the estimators, Bayes risk and posterior risk criteria are used. These point predictors are compared in the sense of their mean squared prediction errors. The average interval length and coverage probability are computed and compared to evaluate the prediction intervals for both sampling schemes. In the present study, the hyperparameters are estimated in two methods. By studying the simulation and presenting real data, the estimation methods are compared, and the performance of the introduced schemes is evaluated.
\end{abstract}

Keywords: Estimation, Prediction, Record, Empirical Bayes, Record ranked set sampling.

Mathematics Subject Classification (2010): 62G30, 62G25, 62C12. 